\title{
Signal, noise power spectrum, and detective quantum efficiency of indirect-detection flat-panel imagers for diagnostic radiology
}

\author{
J. H. Siewerdsen, ${ }^{\text {a) }}$ L. E. Antonuk, Y. El-Mohri, J. Yorkston, and W. Huang
}

Department of Radiation Oncology, University of Michigan Medical Center, Ann Arbor, Michigan 48109

\author{
I. A. Cunningham \\ Imaging Research Laboratories, The John P. Robarts Research Institute and Department of Diagnostic \\ Radiology, London Health Sciences Centre-Victoria, and The University of Western Ontario London, \\ Ontario N6A 5K8, Canada
}

(Received 2 September 1997; accepted for publication 19 February 1998)

\begin{abstract}
The performance of an indirect-detection, active matrix flat-panel imager (FPI) at diagnostic energies is reported in terms of measured and theoretical signal size, noise power spectrum (NPS), and detective quantum efficiency (DQE). Based upon a 1536×1920 pixel, $127 \mu \mathrm{m}$ pitch array of $a$-Si:H thin-film transistors and photodiodes, the FPI was developed as a prototype for examination of the potential of flat-panel technology in diagnostic $x$-ray imaging. The signal size per unit exposure (x-ray sensitivity) was measured for the FPI incorporating five commercially available $\mathrm{Gd}_{2} \mathrm{O}_{2} \mathrm{~S}: \mathrm{Tb}$ converting screens at energies $70-120 \mathrm{kVp}$. One-dimensional and two-dimensional NPS and DQE were measured for the FPI incorporating three such converters and as a function of the incident exposure. The measurements support the hypothesis that FPIs have significant potential for application in diagnostic radiology. A cascaded systems model that has shown good agreement with measured individual pixel signal and noise properties is employed to describe the performance of various FPI designs and configurations under a variety of diagnostic imaging conditions. Theoretical x-ray sensitivity, NPS, and DQE are compared to empirical results, and good agreement is observed in each case. The model is used to describe the potential performance of FPIs incorporating a recently developed, enhanced array that is commercially available and has been proposed for testing and application in diagnostic radiography and fluoroscopy. Under conditions corresponding to chest radiography, the analysis suggests that such systems can potentially meet or even exceed the DQE performance of existing technology, such as screen-film and storage phosphor systems; however, under conditions corresponding to general fluoroscopy, the typical exposure per frame is such that the DQE is limited by the total system gain and additive electronic noise. The cascaded systems analysis provides a valuable means of identifying the limiting stages of the imaging system, a tool for system optimization, and a guide for developing strategies of FPI design for various imaging applications. (c) 1998 American Association of Physicists in Medicine. [S0094-2405(98)00605-1]
\end{abstract}

Key words: digital x-ray imaging, flat-panel imager, amorphous silicon, noise power spectrum, detective quantum efficiency, cascaded systems analysis

\section{INTRODUCTION}

Recently, digital x-ray imagers based upon active matrix, thin-film electronics have emerged as a promising technology for application in diagnostic radiology. Such flat-panel imagers (FPIs) typically incorporate an array of hydrogenated amorphous silicon ( $a$-Si:H) thin-film transistors (TFTs) as pixel switching elements and can detect incident $\mathrm{x}$ rays either directly (by means of a continuous photoconductive layer) or indirectly (by means of a scintillator coupled to a photosensitive pixel element). This paper examines the performance of indirect-detection FPIs, where each pixel is composed of an $a$-Si:H TFT coupled to an optically sensitive $a$-Si:H photodiode. Research into the application of such devices in a variety of imaging fields (e.g., document scanning, ${ }^{1} \mathrm{x}$-ray crystallography, ${ }^{2}$ attenuation correction for emission tomography, ${ }^{3}$ relative dosimetry, ${ }^{4}$ and radiotherapy portal imaging $\left.{ }^{5,6}\right)$ is ongoing, and their potential performance in the field of diagnostic radiology is a subject of considerable interest.

The structure and operation of the FPI under investigation has been detailed extensively, ${ }^{7}$ and only a brief description is given here. Incident $\mathrm{x}$ rays are converted to optical photons in an overlying $\mathrm{x}$-ray converter (typically a luminescent phosphor or CsI:T1), and these quanta are subsequently converted to electron-hole pairs within the $a$-Si:H photodiodes. The image signal is integrated by the photodiodes while the associated TFTs are held in a nonconducting state, and the array is read out by sequentially switching rows of TFTs to a conducting state by means of TFT gate control circuitry. When a row of pixels is switched to a conducting state, charge from those pixels is transferred along data lines and integrated by external charge-sensitive amplifiers. The row is then switched back to a nonconducting state, and the process 
is repeated for each row until the entire array has been read out. The FPI is capable of both single-shot (radiographic) and continuous (fluoroscopic) image acquisition.

The signal and noise performance of such an imaging system may be quantified in terms of observer-independent performance variables (OIPVs). The signal performance of the imaging system can be quantitatively described by the pixel signal size per unit exposure (x-ray sensitivity) and the modulation transfer function (MTF). The noise transfer properties of the imaging system are given by the noise power spectrum (NPS), and the corresponding signal-to-noise performance of the system is described by the detective quantum efficiency (DQE). This paper examines the performance of indirect-detection FPIs through empirical measurement and theoretical modeling of the x-ray sensitivity, NPS, and DQE; determination of the MTF is the subject of other work $^{8,9}$ and is consistent with the results presented herein.

The signal and noise transfer properties of the imaging system can be theoretically described by a cascaded linear systems model, ${ }^{10}$ which allows prediction of the imager signal and noise properties and provides a means of exploring the potential performance of hypothetical FPI designs. A previous article ${ }^{10}$ describes the cascaded systems model in detail, demonstrates its accuracy in describing individual pixel signal and noise measurements, and calculates the imager $\mathrm{DQE}$ as a function of incident exposure, additive electronic noise, and fill factor for conditions corresponding to chest radiography, fluoroscopy, and mammography. In this paper, theoretical calculations are compared to empirical x-ray sensitivity, NPS, and DQE, and the model is used to examine the potential performance of FPIs, incorporating a recently developed, commercially available array proposed for testing and application in diagnostic radiography and fluoroscopy.

\section{METHODS AND MATERIALS}

\section{A. Experimental setup}

Measurements of x-ray sensitivity and NPS were performed using the prototype FPI and x-ray source shown in Fig. 1. The FPI consists of an $a-\mathrm{Si}: \mathrm{H}$ imaging array in combination with an overlying x-ray converter, a dedicated system of acquisition electronics, and a host computer. The array comprises a two-dimensional matrix (1536 $\times 1920$ pixels at $127 \mu \mathrm{m}$ pitch) of $a$-Si:H photodiodes and TFTs, the design and basic performance of which have been previously reported. ${ }^{7}$ Measurements were performed for the array in combination with a variety of commercially available $\mathrm{Gd}_{2} \mathrm{O}_{2} \mathrm{~S}: \mathrm{Tb}$ converters, including Lanex Fast-B $\left(\sim 133 \mathrm{mg} / \mathrm{cm}^{2}\right)$, Regular $\left(\sim 70 \mathrm{mg} / \mathrm{cm}^{2}\right), \quad$ Medium $\left(\sim 59 \mathrm{mg} / \mathrm{cm}^{2}\right)$, Fast-F $\left(\sim 51 \mathrm{mg} / \mathrm{cm}^{2}\right)$, and Fine $\left(\sim 34 \mathrm{mg} / \mathrm{cm}^{2}\right)$. The acquisition electronics ${ }^{11}$ allowed readout of one-third of the array $(512 \times 1920$ pixels $)$ at a maximum fluoroscopic frame rate of $0.24 \mathrm{fps}$, although smaller regions of the imager could be addressed at higher frame rate. A CAMAC crate provided interface to the host computer (Macintosh 650 with 136 MB RAM).

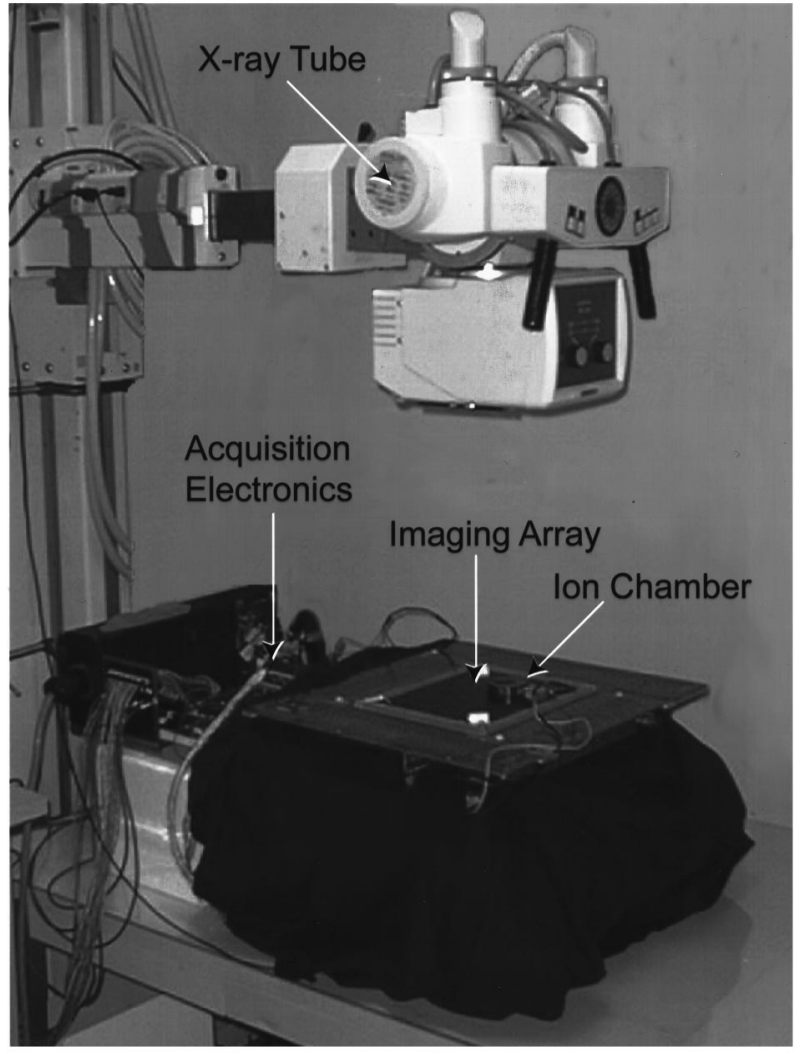

FIG. 1. Experimental setup used in x-ray sensitivity, NPS, and DQE measurements.

The X-ray tube (Dunlee PX1415) was powered by a highfrequency generator (Picker MTX380) in fluoroscopic mode (70-120 kVp, 0-6 mA) and suspended above the imager at variable SID, as shown in Fig. 1. In order to investigate the properties of the imager without introducing various issues of beam quality, measurements reported herein were performed in the absence of a tissue-equivalent phantom; discussion of the effects of beam hardening (e.g., upon signal size and DQE) is given where appropriate. The $\mathrm{kVp}$ of the beam was measured using a calibrated $\mathrm{kVp}$ divider (Keithley $35080 \mathrm{~A}$ with dosimeter $35050 \mathrm{~A}$ ) accurate to $\pm 2 \%$, and the HVL was measured (e.g., $\mathrm{HVL} \sim 4.4 \mathrm{~mm} \mathrm{Al}$ at $90 \mathrm{kVp}$ ) using type $1100 \mathrm{Al}$ HVL filters and a calibrated ion chamber (Keithley 96035). The exposure rate $( \pm 5 \%)$ was measured during data acquisition by placing the ion chamber on the surface of the imager in a region of the x-ray field adjacent to the region being addressed.

\section{B. Empirical $x$-ray sensitivity}

The magnitude of the pixel signal per unit exposure defines the $x$-ray sensitivity, $\Gamma$, of the imager and was measured in a manner reported previously. ${ }^{7}$ Measurements were performed for the FPI employing five Lanex converters at energies between 70 and $120 \mathrm{kVp}$. For a given imager configuration and $\mathrm{kVp}$, the signal from an ensemble of pixels was measured as a function of exposure, and the average of slopes obtained from linear fits to the resulting response data 


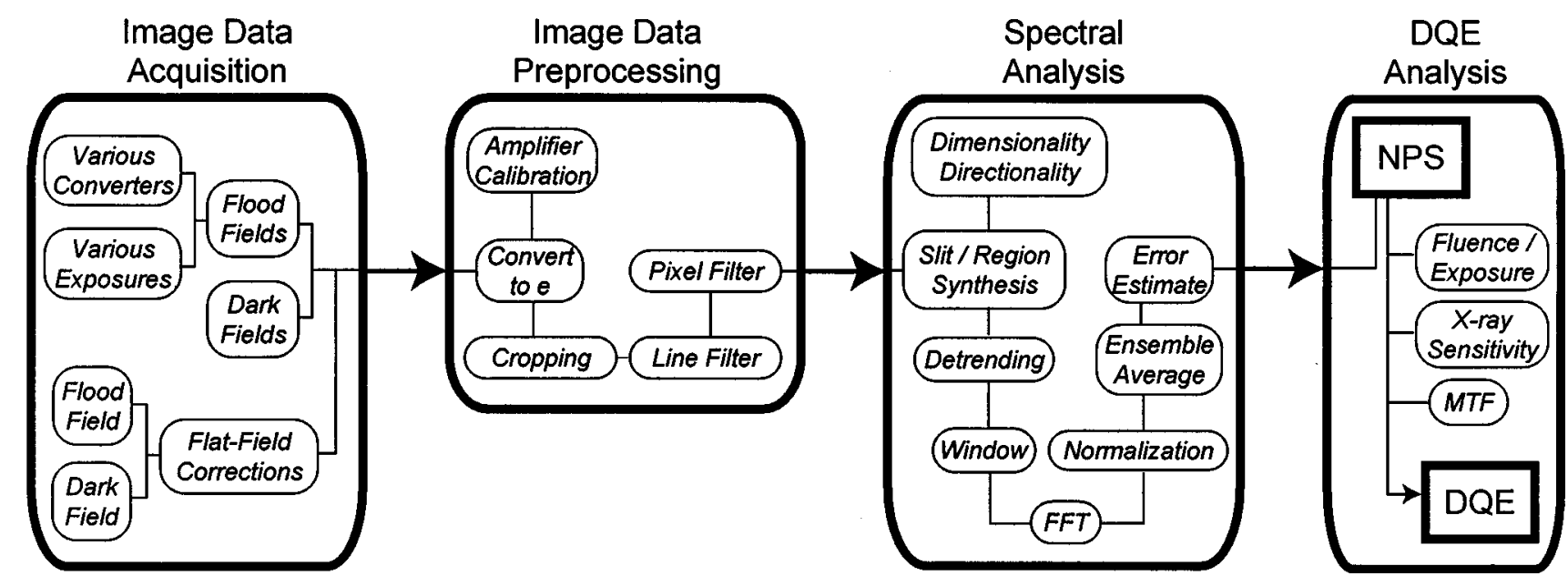

FIG. 2. Block diagram illustrating the methodology for NPS data acquisition, processing, and analysis.

yielded the $\mathrm{x}$-ray sensitivity (units $e / \mathrm{mR} /$ pixel). The SID was fixed at $70 \mathrm{~cm}$, and measurements were performed over a signal range for which the pixel response is known to be highly linear.

\section{Empirical NPS: Measurements and analysis}

NPS measurements were performed for the FPI employing three $\mathrm{Gd}_{2} \mathrm{O}_{2} \mathrm{~S}$ : $\mathrm{Tb}$ converters (Lanex Fast-B, Regular, and Fine) at various exposures up to $\sim 50 \%$ of pixel saturation, where the effects of charge trapping and nonlinearity ${ }^{10}$ are believed to be small. All NPS measurements were performed at $90 \mathrm{kVp}$, with a SID of $\sim 107 \mathrm{~cm}$. The various components and considerations in the NPS measurements and analysis are represented schematically in Fig. 2, including image data acquisition (Sec. II C 1), image data preprocessing (Sec. II C 2), spectral analysis (Sec. II C 3), and determination of the NPS and DQE.

\section{Image data acquisition}

For a variety of x-ray converters and exposures, image data for NPS analysis were acquired, both in the presence ("flood fields") and absence ("dark fields") of x rays. For flood-field acquisition, the fluoroscopic x-ray beam was activated and allowed to stabilize, then the imager was cycled for 30 frames in order to reproducibly initialize the array, and finally, up to eight flood fields were acquired and written to file, each separated by three frames that were discarded. The flood fields form the ensemble for NPS analysis. Due to the relatively slow acquisition electronics and using the lowest stable tube output, the lowest exposure that could be reasonably achieved was $\sim 4 \mathrm{mR}$, which is somewhat higher than typical clinical radiographic exposures (and significantly higher than clinical fluoroscopic exposures). For darkfield acquisition, a similar procedure was followed wherein the x-ray beam was not activated, 30 frames were read out for initialization, and 8 dark fields were acquired, each separated by 3 discarded frames.

For each flood-field measurement, offset and gain (" fflatfield"') corrections were applied to the images in order to correct for stationary variations in pixel response, for variations between the 512 channels of amplifier electronics, and for structure noise inherent to the x-ray converting screen. ${ }^{12}$ The offset and gain corrections for each pixel were derived from 15 averaged dark and flood images obtained prior to data acquisition. The 15 flood images used for the gain corrections were acquired at the same exposure level as the corresponding flood-field measurement.

\section{Image data preprocessing}

The flood fields and dark fields required some preprocessing to yield useful data for NPS analysis. Pixel signal values were converted to units of electrons $(e)$ by means of the measured calibration of the amplifiers. Images were manually cropped to eliminate regions that appeared faulty, typically due to a large number of defective lines or pixels in a given region or to anomalous noise in the acquisition electronics. A $3 \times 3$ median filter was applied to a small number of individual lines that appeared defective. Finally, individual pixel defects were identified by an automated search algorithm, and a $3 \times 3$ median filter was applied. In all cases, defect filtration affected less than $1 \%$ of the total image data and is assumed to have a negligible effect on the NPS measurements.

\section{Spectral analysis and determination of NPS}

Both one-dimensional (1-D) and two-dimensional (2-D) NPS were analyzed from the image data. The 1-D NPS were analyzed by a synthesized slit technique ${ }^{13-15}$ wherein $N$ nonoverlapping slits, each of dimensions $L \times n$ pixels, were selected from the images and summed along the $L$ direction to form $n$-point realizations. For purposes of notation, the horizontal direction on the images - parallel to the TFT control lines-is denoted $x$, with Fourier-pair coordinate $u$; the vertical direction-parallel to the data lines-is denoted by $y$ with Fourier-pair coordinate $\nu$. The 1-D NPS were analyzed with synthesized slits oriented both horizontally [to determine NPS $(u)$ ] and vertically [to determine NPS $(\nu)$ ]. Conver- 
TABLE I. A summary of image data ensembles and the choice of realization dimensions for NPS and DQE analysis.

\begin{tabular}{|c|c|c|c|c|c|c|c|}
\hline $\begin{array}{l}\text { 1-D analysis: } \\
\text { FPI } \\
\text { configuration }\end{array}$ & $\begin{array}{l}\text { Exposure } \\
(\mathrm{mR})\end{array}$ & $\begin{array}{l}\text { Image } \\
\text { format }\end{array}$ & $\begin{array}{l}\text { Image } \\
\text { fields }\end{array}$ & $\begin{array}{c}\text { Slit } \\
(L \times n)\end{array}$ & $\begin{array}{l}\text { Realizations } \\
\qquad(N)\end{array}$ & $\begin{array}{l}\text { NPS } \\
\text { figure }\end{array}$ & $\begin{array}{l}\mathrm{DOE} \\
\text { figure }\end{array}$ \\
\hline Array + Fast-B & 8.4 & $(256 \times 960)$ & 7 & $(40 \times 256)$ & 168 & 6(a) & 9(a) \\
\hline Array + Regular & 8.4 & $(512 \times 1920)$ & 2 & $(40 \times 256)$ & 192 & 6(b) & 9 (b) \\
\hline Array + Fine & 8.4 & $(256 \times 1920)$ & 3 & $(40 \times 256)$ & 144 & 6(c) & $9(\mathrm{c})$ \\
\hline Dark & 0 & $(512 \times 1920)$ & 8 & $(40 \times 256)$ & 768 & 6(d) & $\cdots$ \\
\hline Array+Regular & 4.6 & $(512 \times 960)$ & 5 & $(40 \times 256)$ & 240 & 7(a), (b) & 10 \\
\hline Array+Regular & 6.3 & $(512 \times 960)$ & 5 & $(40 \times 256)$ & 240 & 7(b) & 10 \\
\hline Array + Regular & 8.8 & $(512 \times 960)$ & 5 & $(40 \times 256)$ & 240 & $7(\mathrm{a}),(\mathrm{b})$ & 10 \\
\hline Array + Regular & 10.9 & $(512 \times 960)$ & 5 & $(40 \times 256)$ & 240 & $7(b)$ & 10 \\
\hline Array + Regular & 13.3 & $(512 \times 960)$ & 5 & $(40 \times 256)$ & 240 & 7(a), (b) & 10 \\
\hline $\begin{array}{l}\text { 2-D analysis: } \\
\text { FPI } \\
\text { configuration }\end{array}$ & $\begin{array}{l}\text { Exposure } \\
(\mathrm{mR})\end{array}$ & $\begin{array}{l}\text { Image } \\
\text { format }\end{array}$ & $\begin{array}{l}\text { Image } \\
\text { fields }\end{array}$ & $\begin{array}{l}\text { Region } \\
(m \times n)\end{array}$ & $\begin{array}{c}\text { Realizations } \\
\qquad(N)\end{array}$ & $\begin{array}{l}\text { NPS } \\
\text { figure }\end{array}$ & $\begin{array}{l}\mathrm{DOE} \\
\text { figure }\end{array}$ \\
\hline Array + Fast-B & 8.4 & $(256 \times 960)$ & 7 & $(256 \times 256)$ & 21 & $8(\mathrm{a})$ & $11(\mathrm{a})$ \\
\hline Array + Regular & 8.4 & $(512 \times 1920)$ & 2 & $(256 \times 256)$ & 28 & $8(b)$ & 11(b) \\
\hline Array + Fine & 8.4 & $(256 \times 1920)$ & 3 & $(256 \times 256)$ & 21 & $8(c)$ & $11(\mathrm{c})$ \\
\hline Dark & 0 & $(512 \times 1920)$ & 8 & $(256 \times 256)$ & 112 & $8(d)$ & $\cdots$ \\
\hline
\end{tabular}

gence of the 1-D NPS for various choices of slit length was tested in each case, and it was found that no significant change in the NPS was observed for slit lengths longer than $\sim 0.5 \mathrm{~cm}$, indicating that a central slice of the 2-D NPS was obtained for $L \geqslant 40$. Realizations of width $n=256$ provided a frequency sampling of $0.03 \mathrm{~mm}^{-1}$ and 128 points in the resulting NPS up to the Nyquist frequency $\left(u_{\mathrm{Nyq}}=\nu_{\mathrm{Nyq}}=3.94 \mathrm{~mm}^{-1}\right)$. The 2-D NPS were analyzed separately by selecting regions of dimensions $m \times n$ from the images, giving $N$ 2-D realizations. ${ }^{16}$ Further confirmation of $1-D$ NPS convergence was obtained by comparing the 1-D spectra, with slices of the 2-D spectra near $u=0$ and $\nu=0 .{ }^{16}$ The total number of realizations, $N$, varied depending on the number and dimensions of acquired flood fields and on the amount of cropping necessary in preprocessing. The image data ensembles obtained using various x-ray converters and different exposures and the choice of 1-D and 2-D $L, m, n$, and $N$ are summarized in Table I.

To remove low-frequency background trends from the data, a linear (planar) fit was performed to the 1-D (2-D) realizations and subtracted to yield zero-mean, detrended data. More aggressive detrending (e.g., higher-order fits, or multiple fits to segments of each realization) was examined, but it was found that linear (planar) detrending was sufficient to remove most of the low-frequency trends without affecting the resulting NPS above $\sim 0.03 \mathrm{~mm}^{-1}$. To suppress spectral leakage ${ }^{17}$ (i.e., distortions in the estimated spectra resulting from finite-length realizations), a data window was employed that tapered the realizations near the end points. Several window functions ${ }^{17,18}$ were examined (e.g., Hanning, Hamming, Blackman, and Boxcar), and the differences in the resulting NPS were small. Typically, either a Hanning or Boxcar window was employed.

The $N$ realizations were Fourier transformed using a 1-D (2-D) FFT and normalized according to the choice of slit dimensions ${ }^{13,16}$ and data window ${ }^{17}$ to yield an ensemble of $N$ power spectra, which were averaged to yield the measured NPS. The empirical results were in no way postprocessed or revised based upon theoretical expectations (described below).

\section{Empirical DQE analysis}

From the measured $\Gamma$ and NPS of the imager, the DQE was determined empirically, as described by VanMetter ${ }^{19}$ and Cunningham: ${ }^{20}$

$$
\begin{aligned}
& \operatorname{DQE}(u, \nu) \equiv \frac{\operatorname{NPS}^{\text {deterministc }}(u, \nu)}{\operatorname{NPS}(u, \nu)}, \\
& \operatorname{DQE}(u, \nu)=\frac{\left(\overline{1 / q_{0}}\right)(\Gamma \cdot X)^{2} T_{3}^{2}(u, \nu) T_{5}^{2}(u, \nu)}{\operatorname{NPS}(u, \nu)},
\end{aligned}
$$

where NPS ${ }^{\text {deterministic }}(u, \nu)$ is the NPS expected from an imaging system with deterministic gain and blur (i.e., a system that adds no noise to the image), given by the product of the squares of the system gains and MTFs, and $\operatorname{NPS}(u, \nu)$ is the measured NPS. The incident fluence, $\overline{q_{0}}$, was determined from the measured exposure, $X$, by integrating the known fluence per unit exposure ${ }^{21}$ over the normalized incident $\mathrm{X}$ ray spectrum. ${ }^{22}$ The quantities $T_{3}(u, \nu)$ and $T_{5}(u, \nu)$ are the MTFs of the x-ray converter and photodiode, respectively. $T_{3}(u, \nu)$ was estimated by a Lorentzian fit to empirical data (provided by P. C. Bunch, Ph.D., Eastman Kodak Co.):

$$
T_{3}(u, \nu)=\frac{1}{1+H \cdot\left(u^{2}+\nu^{2}\right)},
$$

where $H$ is a fit parameter. $T_{5}(u, \nu)$ was estimated by a two-dimensional sinc function:

$$
T_{5}(u, \nu)=\left|\operatorname{sinc}\left(a_{\mathrm{pd}} u\right) \cdot \operatorname{sinc}\left(a_{\mathrm{pd}} \nu\right)\right|,
$$

where $a_{\mathrm{pd}}$ is the size of the photodiode aperture, assumed to be square. 
TABLE II. Glossary of terms and symbols relevant to QAD, NPS, and DQE analysis.

\begin{tabular}{|c|c|}
\hline \multicolumn{2}{|c|}{ Cascaded systems analysis: } \\
\hline$i$ & Stage \# in cascaded systems representation of imager \\
\hline$i=0$ & $\mathrm{X}$-ray quanta incident on the imager \\
\hline$i=1$ & Interaction of $\mathrm{x}$ rays in converter \\
\hline$i=2$ & Generation and emission of optical quanta \\
\hline$i=3$ & Spatial spreading of optical quanta \\
\hline$i=4$ & Coupling of optical quanta to detector elements \\
\hline$i=5$ & Integration of optical quanta by photodiodes \\
\hline$i=6$ & Additive electronic noise \\
\hline \multicolumn{2}{|c|}{ Imaging system parameters: } \\
\hline$\overline{q_{0}}$ & Incident $\mathrm{x}$-ray fluence $\left(\mathrm{x}\right.$ rays $\left./ \mathrm{mm}^{2}\right)$ \\
\hline$\underline{X}$ & Exposure $(\mathrm{mR})$ \\
\hline$\overline{g_{1}}$ & Quantum detective efficiency of $x$-ray converter \\
\hline$\overline{g_{2}}$ & Quantum gain of converter \\
\hline$\epsilon_{g 2}$ & Poisson excess in $\overline{g_{2}}$ \\
\hline$T_{3}$ & $\mathrm{X}$-ray converter MTF \\
\hline$\underline{H}$ & Lorentzian fit parameter for $T_{3}$ \\
\hline$\overline{g_{4}}$ & Coupling efficiency of photodiodes \\
\hline$T_{5}$ & Photodiode aperture MTF \\
\hline$a_{p d}$ & Aperture of photodiode (mm) \\
\hline \multicolumn{2}{|c|}{ Empirical and theoretical imager performance parameters: } \\
\hline$(x, y)$ & Spatial coordinates $(\mathrm{mm})$ \\
\hline$(u, v)$ & Spatial frequency coordinates $\left(\mathrm{mm}^{-1}\right)$ \\
\hline$\Gamma$ & Imager $\mathrm{x}$-ray sensitivity $(e / \mathrm{mR} /$ pixel $)$ \\
\hline$L$ & Length of synthesized slit for 1-D NPS analysis \\
\hline$m$ & Length of realizations for 2-D NPS analysis \\
\hline$n$ & Width of realizations for NPS analysis \\
\hline$N$ & Number of realizations for NPS analysis \\
\hline$S_{5}$ & NPS of the presampling signal $\left(\mathrm{mm}^{2}\right)$ \\
\hline$S_{5}^{\dagger}$ & Aliased form of the presampling NPS $\left(\mathrm{mm}^{2}\right)$ \\
\hline$S_{\text {add }}$ & NPS of additive noise sources $\left(\mathrm{mm}^{2}\right)$ \\
\hline$S_{6}$ & Sum of $S_{5}^{\dagger}$ and $S_{\text {add }}\left(\mathrm{mm}^{2}\right)$ \\
\hline MTF & Modulation transfer function \\
\hline NPS & Noise power spectrum \\
\hline $\mathrm{DQE}$ & Detective quantum efficiency \\
\hline
\end{tabular}

\section{E. Cascaded systems analysis}

The flat-panel imaging system can be modeled as a cascade of linear systems, or stages, where each stage represents a physical process possessing its own gain, noise, and spatial spreading properties. ${ }^{10}$ The signal and noise transfer properties of the imager are determined by the transfer properties of the gain and spreading stages, as described by Rabbani et al. ${ }^{23}$ where each stage number, $i$, is characterized by the mean fluence of image quanta, $\overline{q_{i}}$, the mean gain, $\overline{g_{i}}$, the Poisson excess in the gain, $\epsilon_{g i}$, and the MTF, $T_{i}(u, \nu)$. A cascaded systems analysis of the FPI under investigation has been reported previously ${ }^{10,24}$ and was shown to be in general agreement with individual pixel signal and noise measurements. A glossary of terms and symbols used in the analysis (and consistent with the analysis of Ref. 10) is provided in Table II, and a summary of calculated imaging system parameters is given in Table III.

The propagation of image quanta through the system can be represented schematically in a spatial frequencydependent quantum accounting diagram (QAD) ${ }^{25}$ which plots the running product of the gains and squared MTFs as a function of stage number in the imaging chain. The QAD is a simple tool for visualizing the processes and stages governing system performance, and the magnitude of the QAD,

$$
\operatorname{QAD}_{i}(u, \nu)=\prod_{k=1}^{i} \overline{g_{k}} T_{k}^{2}(u, \nu)
$$

is useful in identifying at which stages and at what spatial frequencies quantum sinks occur. ${ }^{26}$ Figure 3(a) shows three QADs for the FPI, employing a Lanex Fast-B, Regular, or Fine converter. The system employing Lanex Fast-B has high intrinsic gain (as evident in the magnitude of the $u$ $=0 \mathrm{~mm}^{-1}$ plot), but suffers at high spatial frequencies due to poor MTF. Alternatively, the system employing Lanex Fine has lower gain (and correspondingly lower QAD at $u$ $=0 \mathrm{~mm}^{-1}$ ) but superior MTF (giving improved QAD at high frequency). The trends demonstrated in these plots are evident throughout the results of this paper, where the continual tradeoffs between system gain and MTF are manifest in the spatial frequency-dependent NPS and DQE.

Alternatively, the DQE may be plotted as a function of the stage number in the imaging chain in order to examine the relative effect of each stage on the image signal-to-noise ratio (SNR). As detailed by Cunningham et al. ${ }^{25}$ the DQE at stage $i$ may be written as

$$
\begin{aligned}
& \operatorname{DQE}_{i}(u, \nu) \\
& \quad=\left[1+\sum_{j=1}^{i}\left(\frac{1+\epsilon_{g_{j}} T_{j}^{2}(u, \nu)+S_{\text {add }_{j}}(u, \nu) / \overline{q_{j}}}{\operatorname{QAD}_{j}(u, \nu)}\right)\right]^{-1} .
\end{aligned}
$$

Whereas the QAD involves only system gains and MTFs, the DQE includes additional terms that affect system performance such as the incident exposure, Poisson excess, and additive electronic noise. Figure 3(b) shows the DQE as a function of stage for the FPI employing a Lanex Fast-B, Regular, or Fine converter. These plots show the dominance

TABLE III. Summary of calculated imaging system parameters.

\begin{tabular}{l|ccccc|cc}
\hline \hline & \multicolumn{5}{|c|}{$\mathrm{Gd}_{2} \mathrm{O}_{2} \mathrm{~S}: \mathrm{Tb}$ converters } \\
$(90 \mathrm{kVp})$ & & \multicolumn{2}{c}{ CsI:Tl converters } \\
Parameter & Fine & Medium & Regular & Fast-F & Fast-B & $250 \mathrm{mg} / \mathrm{cm}^{2}$ & $150 \mathrm{mg} / \mathrm{cm}^{2}$ \\
\hline$\overline{g_{1}}$ & 0.28 & 0.42 & 0.47 & 0.38 & 0.67 & 0.85 & 0.84 \\
$\overline{g_{2}}$ & 600 & 1010 & 1250 & 1470 & 1420 & 610 & 800 \\
$\epsilon_{g 2}$ & 410 & 420 & 470 & 450 & 510 & 110 & 140 \\
$H$ & 0.06 & $\ldots$ & 0.43 & $\ldots$ & 1.25 & $\ldots$ & $\ldots$ \\
$\bar{g}$ & 0.75 & 0.75 & 0.75 & 0.75 & 0.75 & 0.65 & 0.65 \\
\hline \hline
\end{tabular}



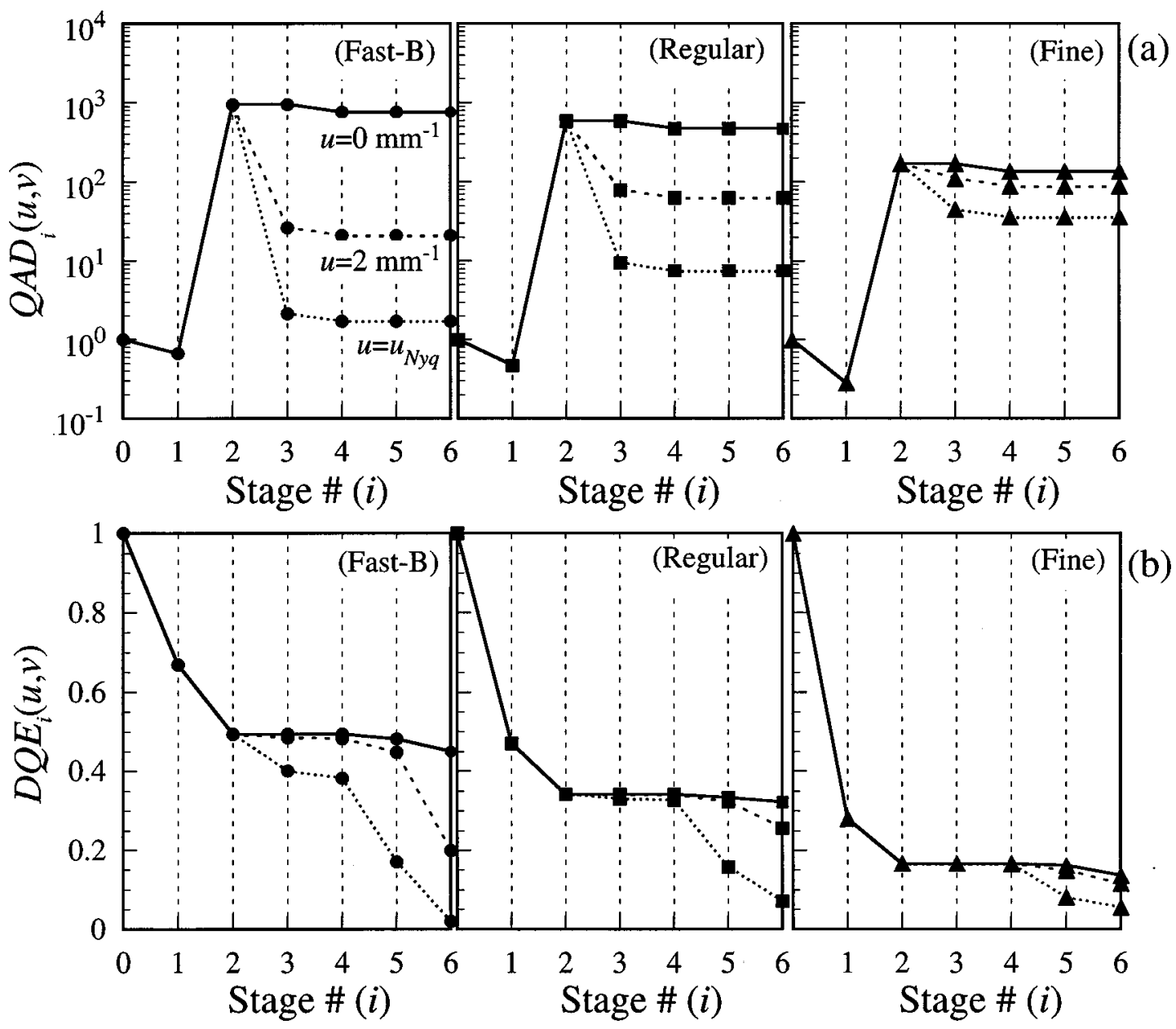

FIG. 3. (a) Quantum accounting diagrams and (b) DQE diagrams for three FPI configurations employed in NPS and DQE measurements. A description of the various stages in the imaging chain and associated notation for system parameters are given in the glossary of Table II. The (Lanex) converters assumed in each case are denoted parenthetically, and the three curves in each plot correspond to different spatial frequencies, $u$, up to the Nyquist frequency, $u_{\mathrm{Nyq}} \cong 3.94 \mathrm{~mm}^{-1}$.

of the primary quantum sink at stage 1 -absorption of incident X-ray quanta-as evident in the QADs, but they also demonstrate the significant effects of the Poisson excess (conversion noise) at stage 2, aliasing of the NPS (included at stage 5, as discussed below in Sec. II E 2), and additive electronic noise [included at stage 6 as a deterministic, unity gain stage with $S_{\text {add }}(u, \nu)$ determined empirically as described above].

\section{Theoretical $x$-ray sensitivity}

The x-ray sensitivity of the imaging pixels is given by a linear combination of the system gains:

$$
\left.\Gamma=\left(\overline{\frac{q_{0}}{X}}\right) a_{\mathrm{pd}}^{2} \overline{g_{1}} \overline{g_{2}} \overline{g_{4}} \text { (units: } e / \mathrm{mR} / \mathrm{pixel}\right) .
$$

Such analysis has demonstrated good agreement with empirical results ${ }^{10}$ for a variety of $\mathrm{x}$-ray converters, pixel designs, and incident $\mathrm{x}$-ray spectra. This paper presents theoretical calculations of imager $\Gamma$ in comparison to measurements obtained using a wider variety of $\mathrm{x}$-ray converters than previously reported ${ }^{10}$ and using the dedicated $\mathrm{x}$-ray source described in Sec. II A.

\section{Theoretical NPS}

The NPS at the presampling stage can be expressed in terms of the system parameters as derived in Ref. 10:

$$
\begin{aligned}
S_{5}(u, \nu)= & a_{\mathrm{pd}}^{4} \overline{q_{0}} \overline{g_{1}} \overline{g_{2}} \overline{g_{4}}\left[1+\overline{g_{4}}\left(\overline{g_{2}}\right.\right. \\
& \left.\left.\left.+\epsilon_{g 2}\right) T_{3}^{2}(u, \nu)\right] T_{5}^{2}(u, \nu) \quad \text { (units: } \mathrm{mm}^{2}\right) .
\end{aligned}
$$

The process of sampling is represented as a multiplication (in the spatial domain) of the presampling detector signal with the sampling grid; hence, as described by Cunningham, ${ }^{27}$ the associated presampling NPS is convolved (in the spatialfrequency domain) with the Fourier transform of the sampling grid to yield

$$
\begin{aligned}
S_{5}^{\dagger}(u, \nu)= & S_{5}(u, \nu) * * \sum_{k, l=-\infty}^{\infty} \delta\left(u-k u_{s}, \nu\right. \\
& \left.-l \nu_{s}\right) \quad\left(\text { units: } \mathrm{mm}^{2}\right),
\end{aligned}
$$

where the $\delta$ functions represent a 2-D comb function with sampling frequencies $u_{s}$ and $\nu_{s}$ determined by the pixel 
pitch. Thus $S_{5}^{\dagger}(u, \nu)$ includes the effects of aliasing on the NPS. Finally, the NPS associated with additive electronic noise is included by addition of the dark-field NPS, $S_{\text {add }}(u, \nu)$, measured in the absence of x-ray irradiation (Sec. II C):

$$
S_{6}(u, \nu)=S_{5}^{\dagger}(u, \nu)+S_{\text {add }}(u, \nu) .
$$

The quantity $S_{\text {add }}(u, \nu)$ includes the effects of pixel dark noise, amplifier noise, residual structure noise, and correlated noise, and is taken as empirical input to the model. The quantity $S_{6}(u, \nu)$ is compared to the measurements described in Sec. II C. The theoretical results were in no way normalized or revised according to the empirical results (described above).

\section{Theoretical DQE}

Using the definition in Eq. (1a), the DQE may be expressed as the ratio of ideal and actual NPS:

$$
\operatorname{DQE}(u, \nu)=\frac{a_{\mathrm{pd}}^{4} \overline{q_{0}}\left[\overline{g_{1}} \overline{g_{2}} \overline{g_{4}} T_{3}(u, \nu) T_{5}(u, \nu)\right]^{2}}{S_{6}(u, \nu)} .
$$

This representation is equivalent to that derived in Ref. 10, which defined the DQE as the ratio of the squares of the output and input frequency-dependent SNR, except that Eq. (8) includes the effect of aliasing of the NPS. The measured [Eq. (1b)] and theoretical [Eq. (8)] DQE are compared for the FPI employing a variety of converters at various exposure levels.

\section{Theoretical performance of FPIs in radiography and fluoroscopy}

A powerful application of the cascaded systems analysis is in describing the potential performance of FPI configurations for various applications. For example, the effect of exposure, pixel fill factor, and additive amplifier noise on the DQE for a wide variety of hypothetical FPI designs was calculated in Ref. 10 for conditions corresponding to chest radiography, general fluoroscopy, and mammography. Recently, indirect-detection flat-panel imaging arrays designed for testing and eventual application in diagnostic radiology have started to become commercially available. Among these is an enhanced $1536 \times 1920$ pixel, $127 \mu \mathrm{m}$ pitch array with a fill factor of $\sim 55 \% .{ }^{28}$ This device is being tested for application in the fields of general radiography and fluoroscopy, and it is interesting to quantitatively examine its potential imaging performance using cascaded systems analysis. This paper examines the DQE calculated as a function of spatial frequency and exposure for FPIs incorporating the enhanced array in combination with either a Lanex Regular phosphor or CsI:T1 scintillator under conditions of chest radiography $(110 \mathrm{kVp} ; \sim 0.03-3 \mathrm{mR})^{29}$ and fluoroscopy (80 $\mathrm{kVp} ; \sim 0.0001-0.01 \mathrm{mR}) .^{30}$

The DQE was calculated as in Eq. (8), except that the denominator $\left[S_{6}(u, \nu)\right]$ was taken to be the sum of the presampling NPS, $S_{5}(u, \nu)$, and the additive electronic NPS, determined solely by the intrinsic pixel noise ${ }^{10}$ and the additive amplifier noise (estimated optimistically to be $\sim 1000 e$ ).
Thus, the calculations include the effects of exposure, choice of x-ray converter, pixel design, and associated electronics, but neglect the effects of residual structure noise and aliasing. Residual structure noise will generally degrade the DQE, but can be minimized through flat-field correction, prudent electronics design, and correlated double sampling. Similarly, aliasing of the NPS will generally degrade the DQE particularly at high spatial frequencies, but for indirectdetection FPIs calculation of the DQE at the presampling stage is usually sufficient to convey the most important aspects of imager performance. ${ }^{27}$

The DQE was calculated for FPIs incorporating the enhanced array operated at full resolution (127 $\mu \mathrm{m}$ pitch) under conditions corresponding to chest radiography and at half-resolution ${ }^{10}$ (254 $\mu \mathrm{m}$ pitch) for general fluoroscopy. For the FPI employing $\sim 70 \mathrm{mg} / \mathrm{cm}^{2} \mathrm{Gd}_{2} \mathrm{O}_{2} \mathrm{~S}: \mathrm{Tb}$, the gains and MTFs were computed as described above. In addition, $\operatorname{DQE}(u=\nu=0)$ was computed for the FPI employing a CsI:T1 converter: $\sim 250 \mathrm{mg} / \mathrm{cm}^{2}$, as reported in combination with a FPI by Wieczorek et al., ${ }^{31}$ and $\sim 150 \mathrm{mg} / \mathrm{cm}^{2}$, equivalent in thickness to the input phosphor of a commercially available x-ray image intensifier (XRII). ${ }^{32}$ The potential spatial resolution (i.e., the MTF) of such FPIs is largely dependent upon the quality of the scintillator processing; nevertheless, due to the crystalline structure of the scintillator, it is expected that FPIs employing CsI:T1 will afford significant improvement in quantum detective efficiency without a serious tradeoff in MTF. The parameters for the FPI employing CsI:T1 were computed in a manner similar to that outlined above: $\overline{g_{1}}$ was computed by integrating the absorption fraction $^{33}$ over the incident spectrum; $\overline{g_{2}}$ and $\epsilon_{g 2}$ were calculated from results reported in the literature, ${ }^{34-36} \overline{g_{4}}$ was calculated by integrating the photodiode quantum efficiency ${ }^{7,28}$ over the emission spectrum for CsI:T1. Results are summarized in Table III.

\section{RESULTS}

\section{A. X-ray sensitivity}

The measured x-ray sensitivity was compared to calculations performed using Eq. (6). Results are shown in Fig. 4, where the discrete symbols correspond to measurements obtained using different $\mathrm{x}$-ray converters at various $\mathrm{kVp}$, and the lines represent theoretical calculations. The $\mathrm{x}$-ray sensitivity clearly scales with the speed of the $\mathrm{x}$-ray converter. Agreement between empirical and theoretical x-ray sensitivity is good (typically better than 5\%), although the level of agreement is different among the five configurations. Excellent agreement is observed for the imager employing Lanex Fast-B, Regular, Medium and Fast-F, and in the case of Lanex Fine the calculations are close to, but systematically higher than, the measured values. Finally, the shape of the theoretical curves agrees quite well with that of the measured data, indicating that the energy dependence has been modeled well in each case.

The $\Gamma$ and MTF of the imager are critical parameters in deciding upon a system configuration for a given application, 


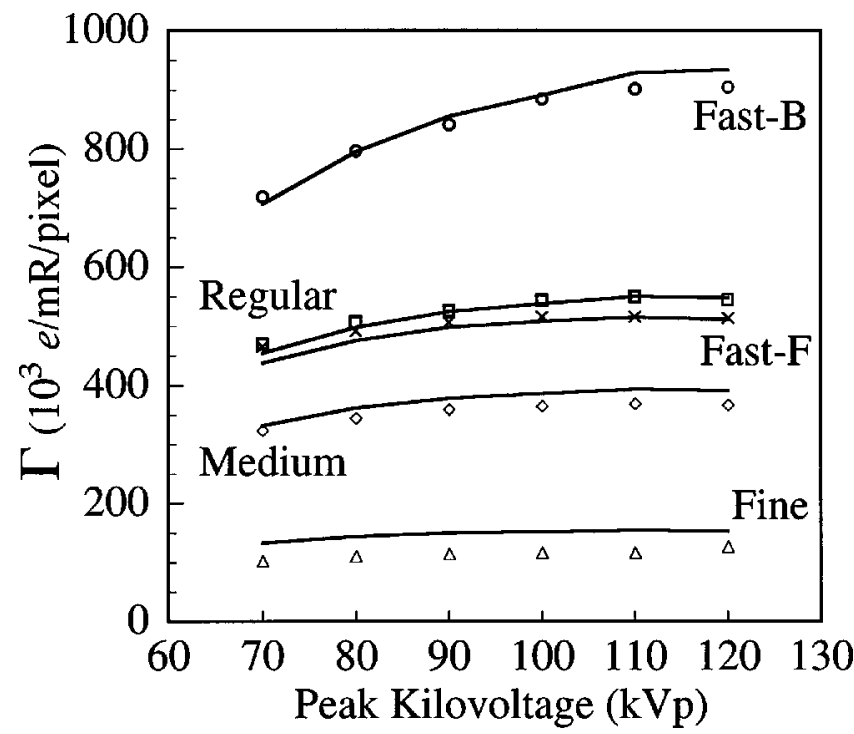

FIG. 4. Measured and theoretical x-ray sensitivity for five configurations of the FPI as a function of $\mathrm{kVp}$. The (Lanex) $\mathrm{x}$-ray converter employed in each configuration is as labeled.

since these quantities determine the signal transfer properties of the imager and are important determinants of imager performance. Knowledge of the magnitude of $\Gamma$ is also important for basic signal considerations, such as the design of the imager amplifier electronics. For example, the charge capacity of the amplifier electronics need not necessarily meet or exceed that of the photodiode; rather, the amplifier should be designed with charge capacity consistent with the expected signal size in a given application, given by the product of $\Gamma$ and the largest expected incident exposure. Of course, the linearity and noise properties of the amplifier, and the charge transients associated with switching the TFT control lines, ${ }^{7}$ should also be taken carefully into account. Furthermore, it should be noted that the results shown in Fig. 4 were obtained for the case of an unattenuated beam, and patient thickness ("beam hardening") certainly affects $\Gamma$. For a harder $\mathrm{x}$-ray beam the quantum detective efficiency $\left(\overline{g_{1}}\right)$ of the converter typically decreases, while the quantum gain $\left(\overline{g_{2}}\right)$ increases. The net result is a slight increase in the x-ray sensitivity. ${ }^{37}$

\section{B. Noise power spectra}

NPS were determined from flood-field images obtained using the FPI employing various x-ray converters at various incident exposures. Figure 5 shows sample $256 \times 256$ realizations obtained at $90 \mathrm{kVp}$ and $\sim 8.4 \mathrm{mR}$ using three Lanex screens [(a), (b), and (c)] and in the dark (d). The gray scale window and level have been adjusted separately in each case in order to maximize contrast. These figures demonstrate the differences in image noise "texture" obtained for different
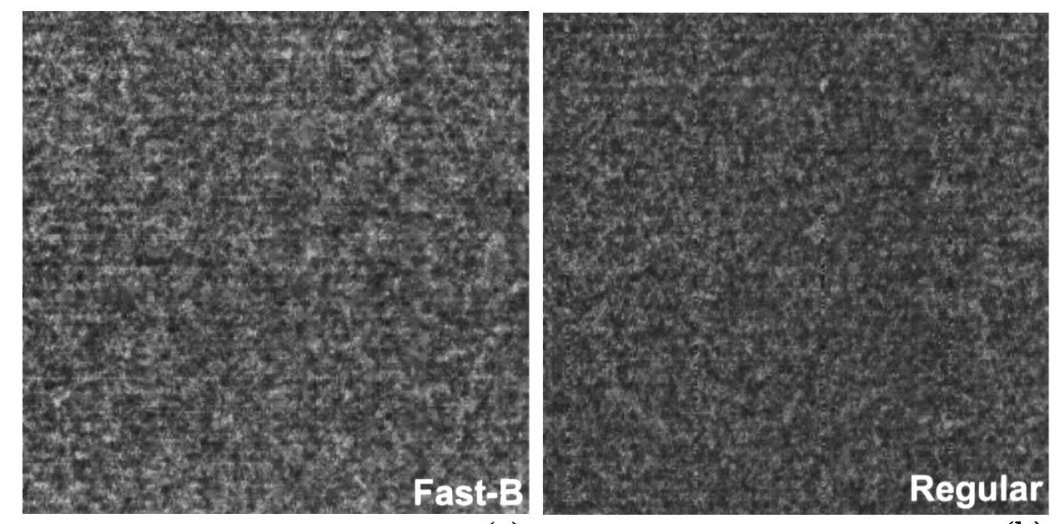

(a)
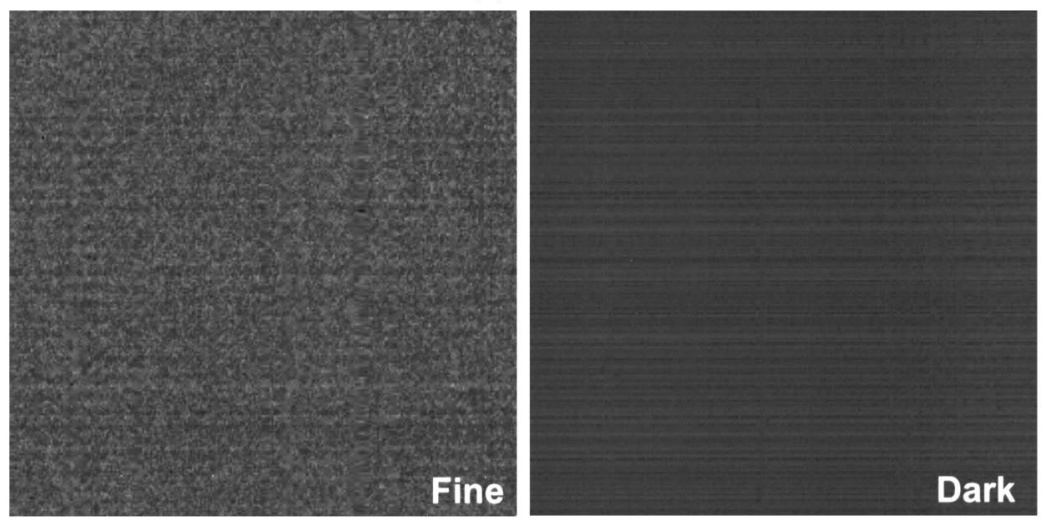

(c)

(d)

FIG. 5. Example $256 \times 256$ realizations obtained for NPS analysis. The images were obtained using the FPI employing (a) Lanex Fast-B, (b) Lanex Regular, (c) Lanex Fine, and (d) in the absence of $x$ rays. 


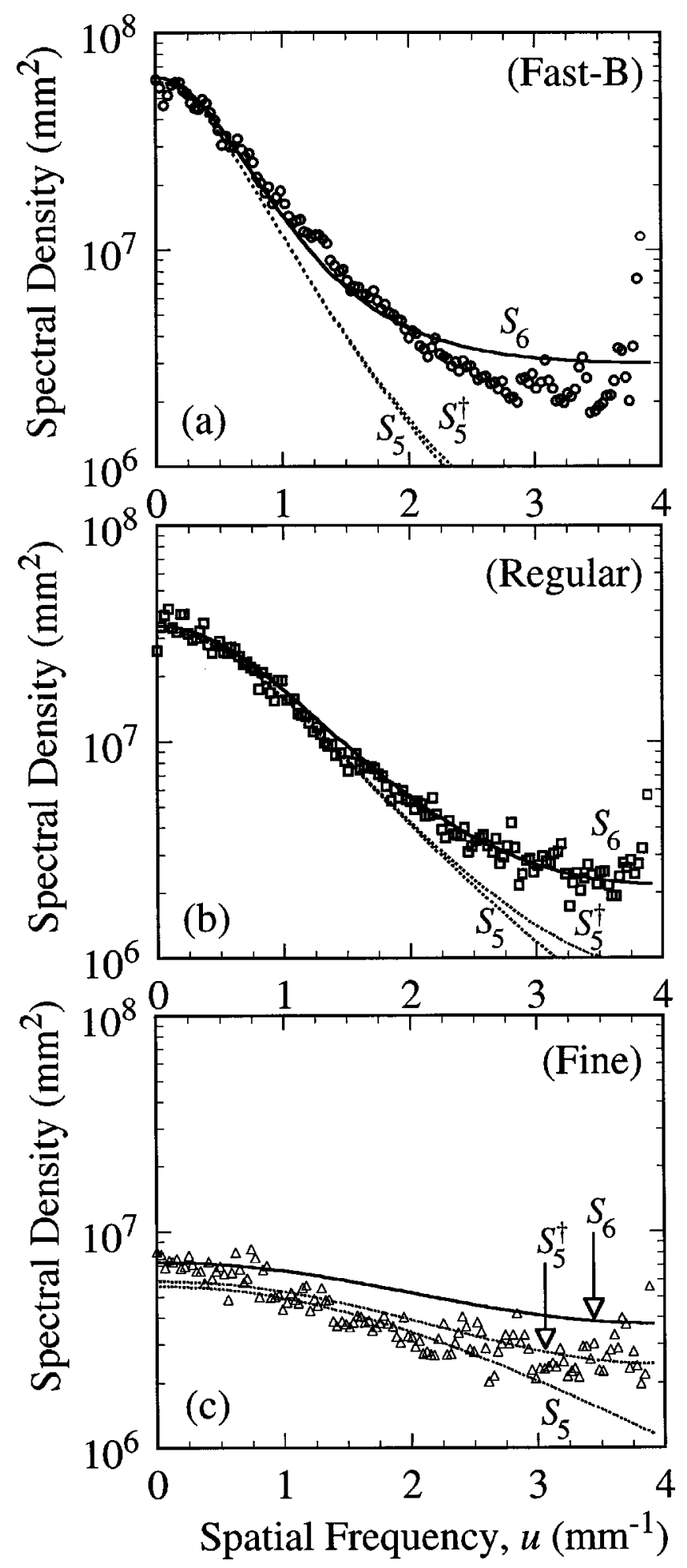

FIG. 6. Empirical and theoretical 1-D NPS $(u)$ for the FPI employing Lanex (a) Fast-B, (b) Regular, and (c) Fine. The theoretical curves in each graph correspond to Eqs. (7a), (7b), and (7c) as labeled and represent the presampling, sampling, and total NPS, respectively.

FPI configurations: for the thickest converter [Fig. 5(a)], image correlations are long range (corresponding to low spatial resolution) and signal fluctuations have a large magnitude (corresponding to high gain); for the thinnest converter [Fig. 5(c)], correlations are short range (corresponding to high
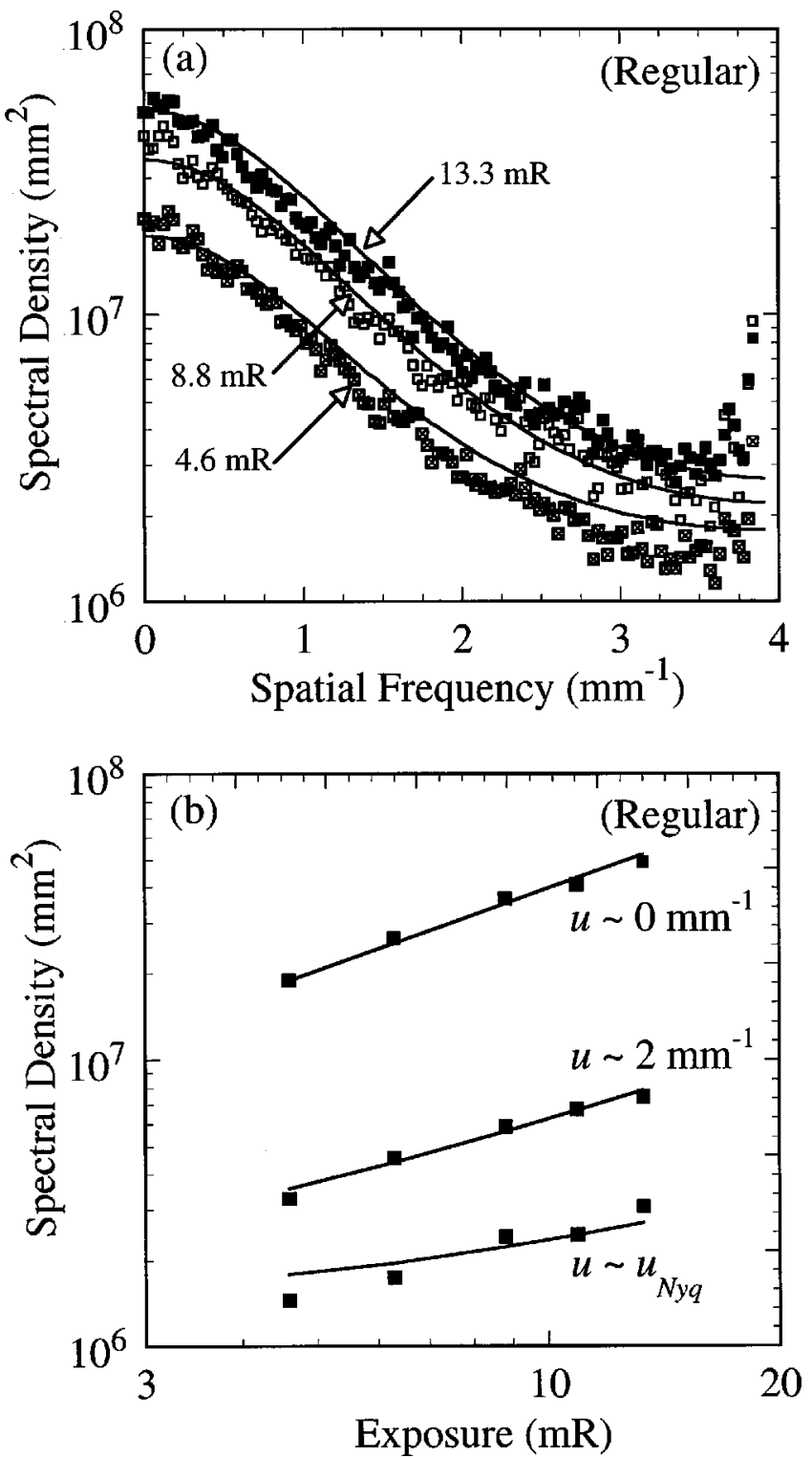

FIG. 7. (a) Empirical and theoretical 1-D NPS( $u$ ) for the FPI employing a Lanex Regular converter at various incident exposures. (b) NPS at various spatial frequencies, $u$, as a function of exposure. In each case, the discrete symbols correspond to empirical results, and the solid curves correspond to theoretical calculations obtained using Eq. (7c).

resolution) and fluctuations are small (corresponding to low gain). In spectral terminology, the image in Fig. 5(a) is "colored," or "red," while the image in Fig. 5(c) is nearly "white." These observations are consistent with expectations based upon the QADs of Fig. 3(a) and are quantified in the NPS. The dark-field realization shown in Fig. 5(d) demonstrates the intrinsic character of the imaging array and associated electronics. A relatively strong horizontal correlation is evident, associated with nonstationary fluctuations arising from the TFT switching electronics. (Stationary vertical variations between amplifier electronics channels have been corrected by the flat-field procedure.) This image suggests that resulting NPS will exhibit a strong correlated component in the $\nu$ direction, as shown below.

The 1-D NPS $(u)$ are shown in Fig. 6 for the FPI employ- 


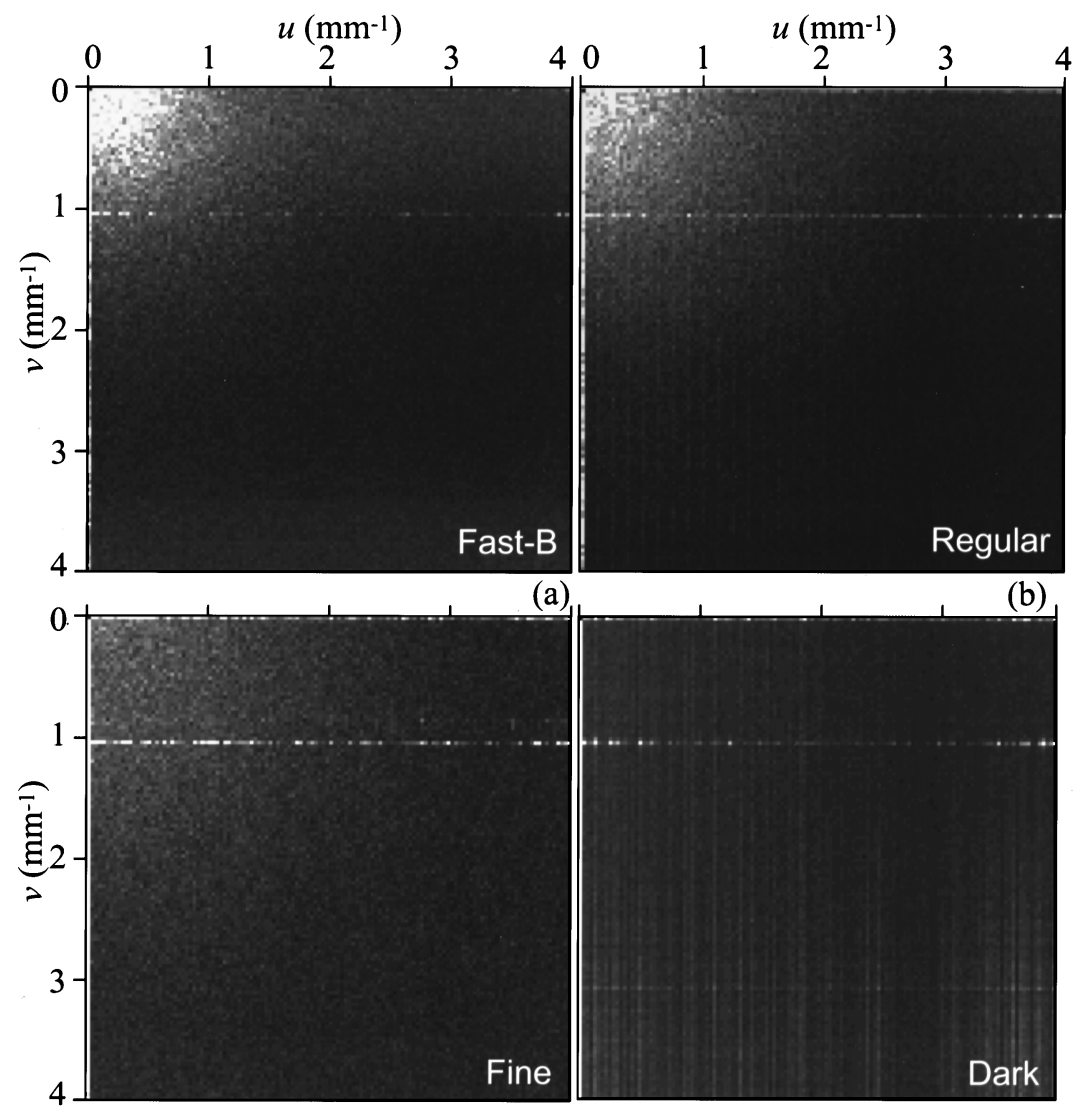

(c)

(d)

FIG. 8. Gray scale representations of 2-D NPS $(u, \nu)$ measured under conditions corresponding to the data realizations of Fig. 5 for the FPI employing (a) Lanex Fast-B, (b) Lanex Regular, (c) Lanex Fine, and (d) in the absence of x rays. The gray scale is such that white (black) corresponds to high (low) power spectral density.

ing three Lanex converters. In each case, the discrete symbols correspond to measurements, and the curves correspond to theoretical calculations performed using Eqs. (7a), (7b), and $(7 \mathrm{c})$. The zero-frequency values of the measured NPS are not plotted. In each case, we observe fairly good agreement in the magnitude (which is determined by the system gains) and shape (which is determined by the system MTFs) of the measured NPS and theoretical predictions. For the FPI using Lanex Fast-B, there is fair agreement throughout, with a slight underestimation (overestimation) of the NPS at middle (high) frequencies. In this case, due to strong bandlimiting of the NPS by the low MTF of the converter, there is little effect of aliasing, and $S_{5}$ and $S_{5}^{\dagger}$ are nearly identical. For the FPI using Lanex Regular, we observe excellent agreement between measured and theoretical results up to $u_{\text {Nyq }}$. For the FPI using Lanex Fine, the measured NPS and the theoretical prediction have a similar shape, but the theory slightly overestimates the magnitude of the NPS in a manner consistent with the overestimation of $\Gamma$, as shown in Fig. 4. Also, there is a more significant effect of aliasing in this case, where the high converter MTF is such that there is a relatively large amount of noise power above the sampling frequency. The "spike" in the measured NPS at $u_{\mathrm{Nyq}}$ is likely due to residual structure between adjacent data lines and/or residual pixel defects.

Figure 7 shows measured NPS and theoretical predictions for the FPI employing Lanex Regular at various incident exposures. Figure 7(a) shows the NPS as a function of spatial frequency for three exposures $(4.6,8.8$, and $13.3 \mathrm{mR})$, and fair agreement is observed between the measurement and theory in each case. Furthermore, the NPS is seen to scale approximately linearly with incident exposure. This is evident in Fig. 7(b), where the power spectral density at various spatial frequencies is plotted as a function of exposure. In each case, we observe proportionality between spectral density and exposure consistent with Eq. (7c).

Figure 8 shows gray scale representations of measured 2-D NPS $(u, \nu)$ corresponding to the conditions of the image realizations in Fig. 5. Such plots are illustrative of off-axis NPS structure that could otherwise be missed in a 1-D analysis. ${ }^{16}$ For the thickest converter [Fig. 8(a)], the NPS exhibits high spectral density at low frequencies (corresponding to high gain) and strong bandlimiting at higher frequencies (corresponding to poor MTF). For the thinnest converter [Fig. 8(c)] we observe low spectral density at low frequencies (due to low gain) that falls off gradually in frequency space (due to high MTF). Each NPS exhibits a strong correlation at $\nu \sim 1 \mathrm{~mm}^{-1}$, associated with the horizontal striations evident in Fig. 5(d). This correlation corresponds to a periodicity of $\sim 8$ lines, which is visually apparent in the flood fields given a sufficiently narrow gray scale window. For the dark-field NPS [Fig. 8(d)], a more subtle correlation 

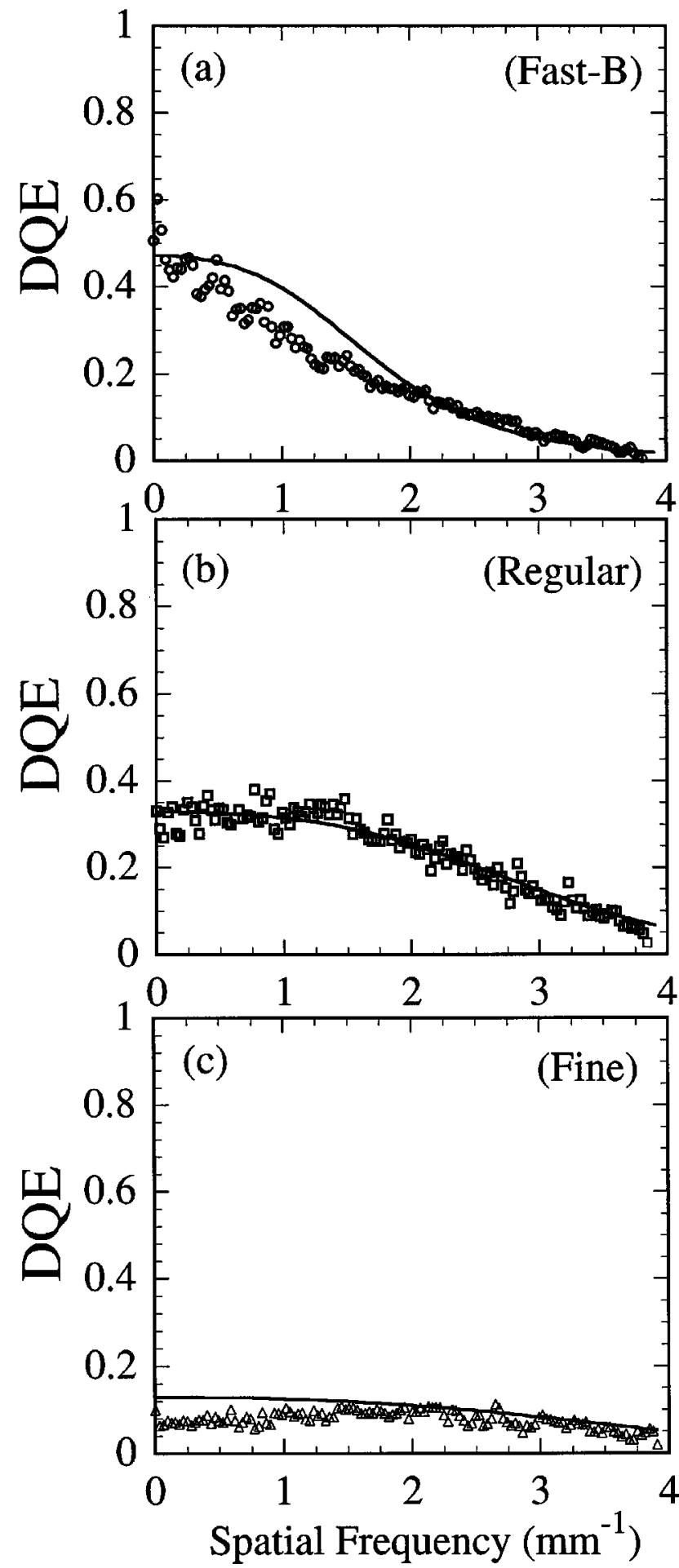

FIG. 9. Empirical and theoretical 1-D DQE $(u)$ corresponding to the NPS of Fig. 6 for the FPI, employing Lanex (a) Fast-B, (b) Regular, and (c) Fine.

is evident at $\nu \sim 3 \mathrm{~mm}^{-1}$, which is dominated by quantum noise in the flood-field spectra. These correlated components of the NPS are caused by nonstationary fluctuations in the TFT switching electronics and can be removed through differential sampling of pixel values from each row with a "dummy" (i.e., optically insensitive) pixel from the same row. As mentioned, the 1-D NPS of Fig. 6 are consistent

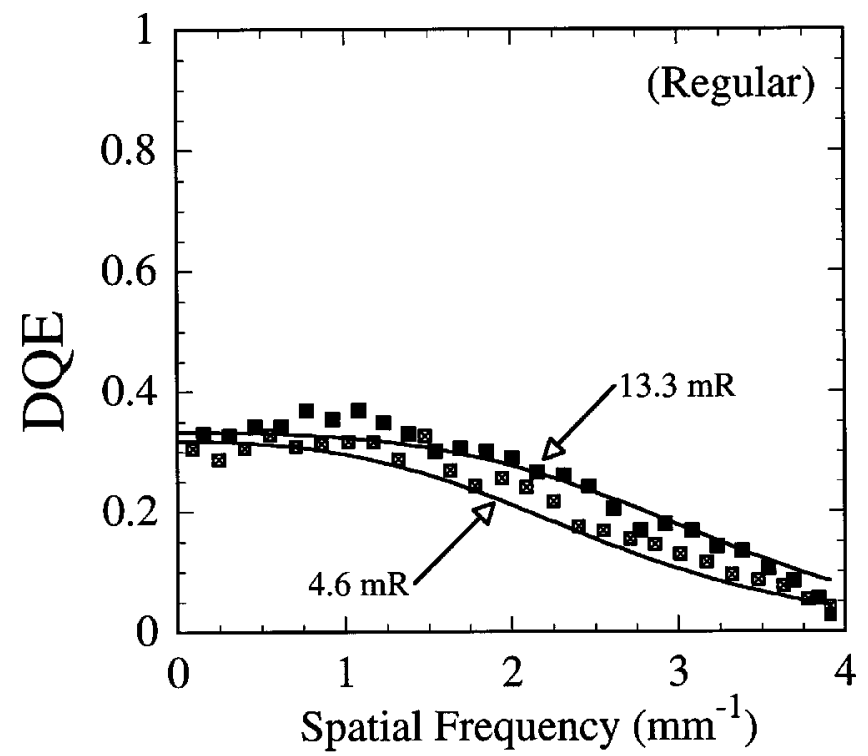

FIG. 10. Empirical and theortical 1-D DQE $(u)$ corresponding to the NPS of Fig. 7 obtained for the FPI employing Lanex Regular at various incident exposures.

with the 2-D NPS of Fig. 8 near the $u$ axis. These spectra clearly demonstrate the continual tradeoffs between converter gain and MTF for powder phosphors, as indicated in the QADs of Fig. 3(a).

\section{Detective quantum efficiency}

The measured DQE was determined using Eq. (1) for the FPI employing various converters and as a function of exposure. From the NPS of Fig. 6, the DQE for the FPI in combination with Lanex Fast-B, Regular, and Fine were determined and are shown in Fig. 9, in comparison with calculations performed using Eq. (8). At the exposures used in these measurements, the DQE in each case is dominated by the quantum detective efficiency of the converter, $\overline{g_{1}}$, and the Poisson excess in the quantum gain, $\epsilon_{g 2}$. For the FPI configuration employing Fast-B, we observe fair agreement between measured and theoretical DQE at low and high frequencies, with some discrepancy at midfrequencies possibly due to a difference between the MTF of the converter used in the NPS measurement and that from which the Lorentzian fit [Eq. (2)] was determined. For the FPI employing Lanex Regular, there is excellent agreement between measured and theoretical DQE across all frequencies. For Lanex Fine, we observe fair agreement, but with a slight discrepancy consistent with the overestimation of $\Gamma$.

From the NPS of Fig. 7, the DQE was determined for the FPI employing Lanex Regular at various exposures and are shown in Fig. 10. DQE is dependent on incident exposure only in proportion to the additive noise; hence, over the range of relatively high exposures that could be examined using the present acquisition system, the exposure has a relatively small effect on DQE. Therefore, for reasons of clarity only results for the highest and lowest exposures are shown. At low spatial frequencies the DQE is dominated by $\overline{g_{1}}$ and 

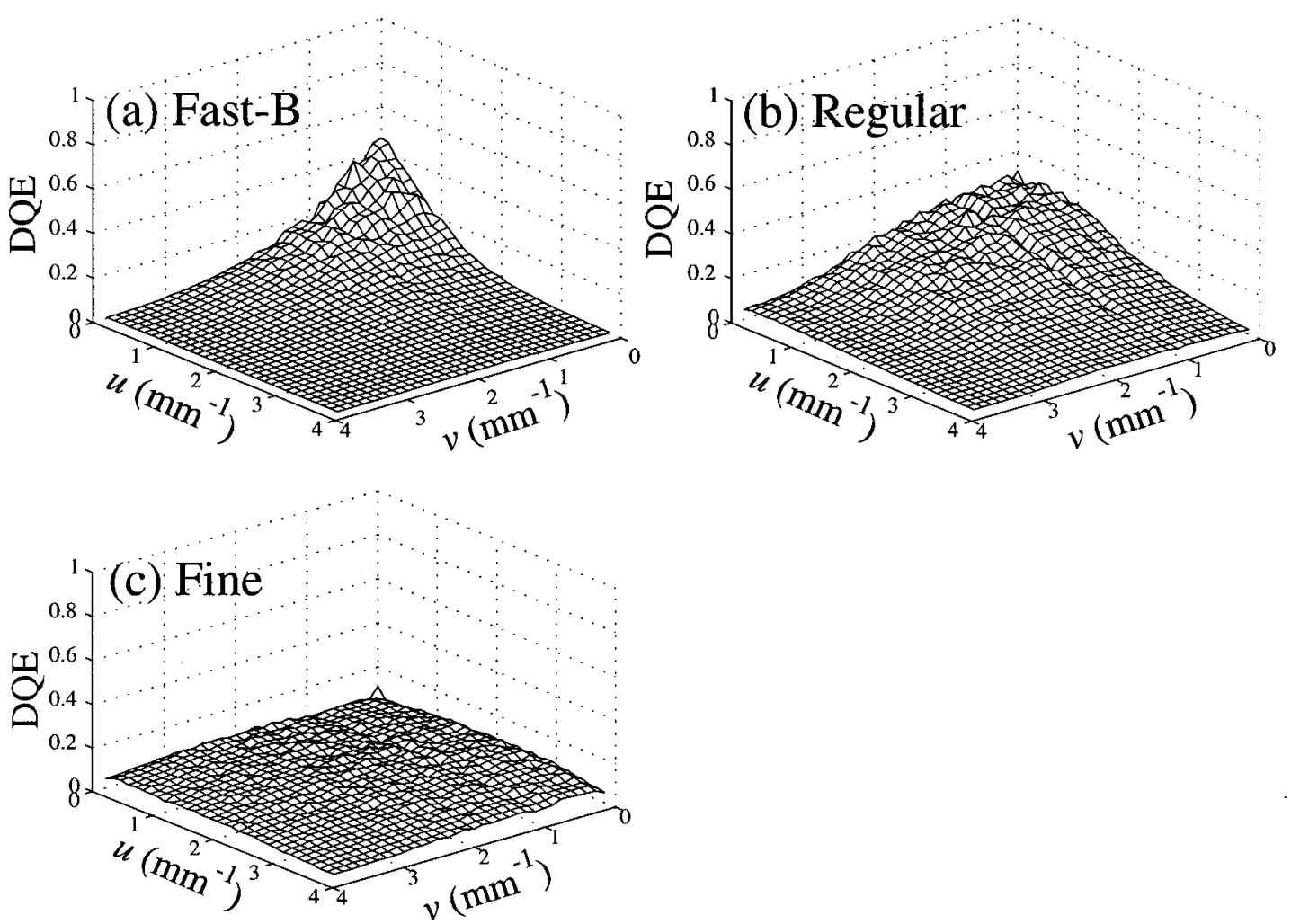

FIG. 11. 2-D DQE $(u, v)$ corresponding to the NPS of Fig. 8 measured for the FPI employing Lanex (a) Fast-B, (b) Regular, and (c) Fine.

$\epsilon_{g_{2}}$, but at high spatial frequencies the exposure level (relative to the additive electronic noise) becomes significant. Once again, there is fair agreement between measured and theoretical DQE. A new system of acquisition electronics being implemented will provide reduced electronics noise and allow an examination of the DQE at lower exposures.

From the 2-D NPS of Fig. 8, the 2-D DQE were analyzed and are presented in Fig. 11. Perhaps the best illustration of the trends described by the QADs of Fig. 3(a) are in the 2-D plots of DQE: for the thickest converter [Fig. 11(a)] the system has high DQE at low frequency (due to high $\bar{g}_{1}$ ) but shows significant degradation at high frequency (due to low MTF); for the thinnest converter [Fig. 11(c)], the DQE is relatively low at low frequency (due to low $\bar{g}_{1}$ ) but is maintained at higher frequency (due to high MTF), where it is, in fact, superior to the DQE of the system employing the thicker phosphor. The correlated noise at $\nu \sim 1 \mathrm{~mm}^{-1}$ causes a degradation in DQE at that frequency.

The effects of beam hardening upon the DQE (e.g., by attenuation of the beam in tissue) depend upon the tradeoffs in quantum detective efficiency, quantum gain, Poisson excess, and MTF. For example, as mentioned above, the value of $\bar{g}_{1}$ generally decreases for a harder beam, whereas the value of $\bar{g}_{2}$ increases; therefore, under conditions where the DQE is limited by $\bar{g}_{1}$ (e.g., at high exposures) the DQE will decrease with beam hardening; conversely, under conditions where the DQE is limited by the optical gain in proportion to the additive noise (i.e., at low exposures and high spatial frequency) the DQE may increase. Thus, the relative effect of each parameter upon DQE for various beam qualities depends in a fairly complicated manner upon the imager con- figuration and exposure conditions, and a complete description of such effects is beyond the scope of this paper.

\section{Theoretical performance of FPIs in radiography and fluoroscopy}

Figure 12(a) shows the DQE calculated for two FPI configurations incorporating the enhanced, commercially available $127 \mu \mathrm{m}$ array. Calculations are shown as a function of spatial frequency over the range of exposures typical of chest radiography. The 2-D surface plot corresponds to the FPI employing $\sim 70 \mathrm{mg} / \mathrm{cm}^{2} \mathrm{Gd}_{2} \mathrm{O}_{2} \mathrm{~S}: \mathrm{Tb}$, and the 1-D curve in the DQE versus $X$ plane corresponds to the zero-frequency DQE computed for the FPI employing $\sim 250 \mathrm{mg} / \mathrm{cm}^{2}$ CsI:Tl. Over the range of typical exposures, the DQE has only a slight dependence on incident exposure, except at high spatial frequencies, where the effect of additive electronic noise becomes significant. Similarly, at the lowest exposures the frequency dependence of the DQE is more severe, again due to the increased effect of additive noise. The FPI employing CsI:Tl is seen to give significant enhancement in quantum detective efficiency, and in each case the DQE is determined primarily by $\bar{g}_{1}$ and $\epsilon_{g 2}$.

Figure 12(b) shows the DQE calculated for conditions corresponding to general fluoroscopy for two FPI configurations incorporating the enhanced array operated at halfresolution and at $\sim 30 \mathrm{fps}$. Over this range of extremely low exposures, the DQE is strongly dependent upon incident exposure, since the additive electronic noise is of the same order as (or much larger than) the incident $\mathrm{x}$-ray fluence. For example, at the lowest exposures per frame, there 
are approximately $\left(2 \times 10^{5} \times\right.$ rays $\left./ \mathrm{mm}^{2} / \mathrm{mR}\right) \times(0.0001 \mathrm{mR})$ $\times(0.254 \mathrm{~mm})^{2}=\sim 1$ incident $\mathrm{x}$ ray per pixel. Considering the system $x$-ray sensitivity $(\sim 50-250 e$ /incident $x$ ray $)$, the requirements upon the level of additive electronic noise consistent with good DQE are extremely challenging. The FPI employing CsI:Tl has improved DQE compared to that employing $\mathrm{Gd}_{2} \mathrm{O}_{2} \mathrm{~S}: \mathrm{Tb}$, but suffers similarly at low exposures. These calculations illustrate the challenging nature of the fluoroscopic application.

\section{DISCUSSION AND CONCLUSIONS}

Measurements of x-ray sensitivity, NPS, and DQE for indirect-detection, active matrix FPIs have been reported and compared to predictions based upon a cascaded linear systems model of such imagers. X-ray sensitivity was measured for the FPI in combination with five commercially available phosphor screens at $70-120 \mathrm{kVp}$. For all configurations and across all energies, the theoretical model provided an accurate prediction of the imager signal (Fig. 4). NPS and DQE were measured for the FPI employing three Lanex screens and at various exposures, and good agreement was observed between empirical and theoretical results (Figs. 6, 7, 9, and $10)$.
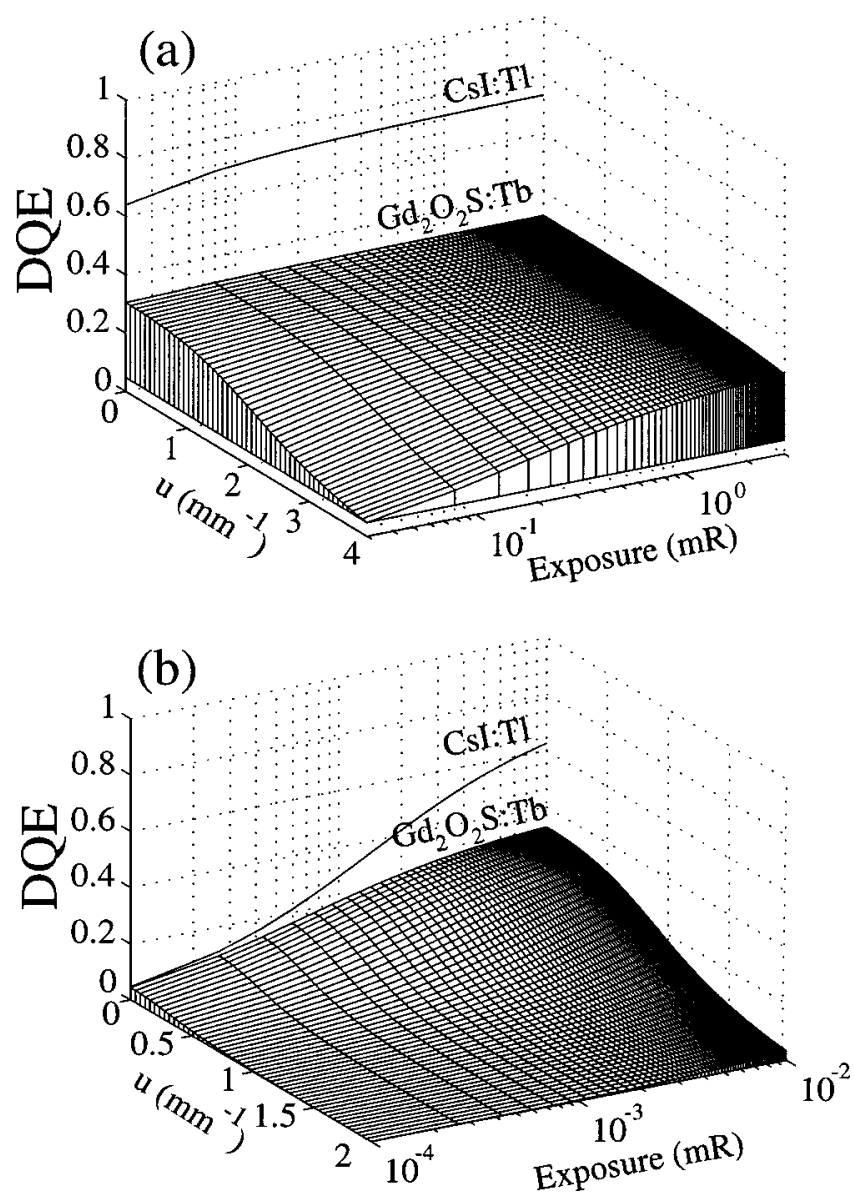

FIG. 12. Theoretical DQE versus spatial frequency $(u)$ and exposure $(X)$ for the enhanced design FPI under conditions corresponding to (a) chest radiography and (b) fluoroscopy.
The $\mathrm{x}$-ray sensitivity, NPS, and DQE constitute a comprehensive, quantitative characterization of the FPI signal and noise performance. The x-ray sensitivity provides useful information regarding the expected signal size for a given FPI configuration and is an essential consideration in deciding upon the requirements of pixel charge capacity, amplifier charge capacity, and tolerable system noise levels. The NPS and DQE provide a quantitative, observer-independent measure of imager performance and allow a degree of objective comparison between the performance of different technologies. For example, the DQE for the indirect-detection FPIs in combination with Lanex Regular is potentially comparable or superior to that of existing radiographic screen-film ${ }^{38,39}$ and computed radiography ${ }^{16}$ systems, and FPIs incorporating a high-quality CsI:Tl converter could offer a significant improvement. Fluoroscopy, however, represents a more challenging application for flat-panel imaging technology due to the extremely low exposure per frame. Although the DQE is limited primarily by the quantum detective efficiency at high exposures, system gain and additive electronic noise become critical parameters at lower exposures, and it remains to be seen whether indirect-detection FPIs can meet or exceed the DQE of modern XRIIs.

FPIs offer a number of significant advantages over existing imaging technology apart from signal and noise considerations. Large area FPIs can be packaged in a compact, thin-profile system and provide real-time, digital image acquisition and display-important attributes in an era of digital radiology and teleradiology. They exhibit images free of geometric (e.g., pin-cushion, barrel, or $S$-wave) distortion, veiling glare, and blooming. Originally developed for application in radiotherapy portal imaging, the imagers possess excellent radiation damage resistance. ${ }^{40,41}$ Furthermore, FPIs offer the potential of both single-image (radiographic) or continuous (fluoroscopic) image acquisition. This could be a particularly valuable feature in clinical environment where the imaging task routinely and rapidly switches between real-time fluoroscopy and radiography (e.g., spot film).

As FPIs become commercially available, it is important to understand the signal and noise performance of such systems for various configurations and applications. Calculations for FPI configurations incorporating a commercially available $127 \mu \mathrm{m}$ pitch, $\sim 55 \%$ fill factor imaging array indicate a strong dependence of the DQE on the choice of x-ray converter and field of application. For chest radiography such FPIs could potentially provide DQE exceeding that of screen-film systems [Fig. 12(a)]. For fluoroscopic imaging, the DQE at low exposures is limited by the quantum detective efficiency and quantum gain of the converter and, especially, the additive electronic noise of the pixel and amplifier [Fig. 12(b)]. Of course, the exposure per frame (and hence the DQE) could be increased by reducing the frame rate, but this could limit the practical applicability and utility of the imager for many fluoroscopic procedures.

Flat-panel imaging technology could eventually offer a useful tool for clinical diagnostic radiology; however, a number of technical challenges remain to be addressed in order to realize its potential. Development of high-quality x-ray con- 
verters, such as thick, high-resolution CsI:Tl, is likely an important step in maximizing the DQE for indirect-detection FPIs. The achievement of larger area arrays (up to 30 $\left.\times 40 \mathrm{~cm}^{2}\right)$ with higher resolution $(\sim 100 \mu \mathrm{m}$ pitch or finer $)$ and higher fill factor $(50 \%-100 \%)$ is generally considered a desirable goal toward the application of FPIs in radiography and, especially, mammography. Acquisition electronics capable of high-speed (e.g., up to $30 \mathrm{fps}$ ), low-noise $(<1000 e)$ readout are desirable, if not necessary, developments toward the application of FPIs in fluoroscopy. FPIs based upon direct detection, such as those employing $a-\mathrm{Se}^{42,43}$ or $\mathrm{PbI}_{2},{ }^{44}$ offer the potential of high quantum detective efficiency and spatial resolution, but face challenges as well. Aliasing of the NPS can significantly degrade the DQE of such systems, ${ }^{45}$ and issues of system gain and additive electronic noise are important considerations for both direct and indirect-detection FPIs at the low exposures typical of fluoroscopy. Ultimately and despite these challenges, however, FPIs represent a highly promising technology for digital x-ray imaging in diagnostic radiology.

\section{ACKNOWLEDGMENTS}

The authors extend their sincere gratitude to D. P. Trauernicht, Ph.D. for many useful discussions regarding the NPS data analysis and to K. W. Jee, M.Sc. and M. Verma, B.E. for assistance with the measurements. We also thank J. M. Boudry, Ph.D. for valuable discussions regarding intrinsic pixel noise and for assistance with the experimental setup. This work is supported by National Institutes of Health Grant No. R01-CA56135.

${ }^{a}$ Corresponding author: Jeffrey H. Siewerdsen, M.Sc., Rm. B2C432, Department of Radiation Oncology, University of Michigan Medical Center, 1500 E. Medical Center Dr., Ann Arbor, Michigan 48109-0010. Electronic mail: jsiewerd@umich.edu

${ }^{1}$ R. A. Street, Hydrogenated Amorphous Silicon (Cambridge U.P., New York, 1991).

${ }^{2}$ S. Ross, I. Naday, M. Kanyo, M. L. Westbrook, E. M. Westbrook, W. C. Phillips, M. J. Stanton, and R. A. Street, "Amorphous silicon area detectors for protein crystallography," Charge Coupled Device and Solid State Optical Sensors V, SPIE 2415, 1995, pp. 189-203.

${ }^{3}$ L. E. Antonuk, J. Boudry, W. Huang, K. L. Lam, E. J. Morton, R. K. Ten Haken, J. Yorkston, and N. H. Clinthorne, "Thin-film, flat-panel, composite imagers for projection and tomographic imaging," IEEE Trans. Med. Imaging 13, 482-490 (1994).

${ }^{4}$ Y. El-Mohri, L. E. Antonuk, J. Yorkston, B. A. Fraass, K. Jee, J. H. Siewerdsen, W. Huang, and V. E. Scarpine, "Relative dosimetry using an active matrix flat-panel imager," abstract and talk given at the 1996 Annual Meeting of the American Association of Physicists in Medicine, July, 1996.

${ }^{5}$ L. E. Antonuk, J. Boudry, W. Huang, D. L. McShan, E. J. Morton, J. Yorkston, M. J. Longo, and R. A. Street, "Demonstration of megavoltage and diagnostic x-ray imaging with hydrogenated amorphous silicon arrays," Med. Phys. 19, 1455-1466 (1992).

${ }^{6}$ L. E. Antonuk, J. Yorkston, W. Huang, H. Sandler, J. H. Siewerdsen, and Y. El-Mohri, "Megavoltage imaging with a large-area, flat-panel, amorphous silicon imager," Int. J. Radiat. Oncol., Biol., Phys. 36, 661-672 (1996).

${ }^{7}$ L. E. Antonuk, Y. El-Mohri, J. H. Siewerdsen, J. Yorkston, W. Huang, and V. E. Scarpine, "Empirical investigation of the signal performance of a high-resolution, indirect detection, active matrix flat panel imager (AMFPI) for diagnostic radiology,"' Med. Phys. 24, 51-70 (1997).

${ }^{8}$ J. Yorkston, L. E. Antonuk, N. Seraji, W. Huang, J. Siewerdsen, and Y. El-Mohri, "Evaluation of the MTF for $a$-Si:H imaging arrays," Medical
Imaging 1994: Physics of Medical Imaging, SPIE 2163, 1994, pp. 141148.

${ }^{9}$ J. Yorkston, L. E. Antonuk, N. Seraji, W. Huang, J. Siewerdsen, and Y. El-Mohri, "MTF measurements with high resolution $a$-Si:H imaging arrays," Medical Imaging 1995: Physics of Medical Imaging, SPIE 2432, 1995, pp. 260-269.

${ }^{10}$ J. H. Siewerdsen, L. E. Antonuk, Y. El-Mohri, J. Yorkston, W. Huang, J. M. Boudry, and I. A. Cunningham, "Empirical and theoretical investigation of of the noise performance of indirect detection, active matrix flatpanel imagers (AMFPIs) for diagnostic radiology," Med. Phys. 24, 71-89 (1997)

${ }^{11}$ E. J. Morton, L. E. Antonuk, J. E. Berry, W. Huang, P. Mody, and J. Yorkston, "A data acquisition system for flat-panel imaging arrays," IEEE Trans. Nucl. Sci. 41, 1150-1154 (1994).

${ }^{12}$ L. E. Antonuk, J. Boudry, W. Huang, D. L. McShan, E. J. Morton, J. Yorkston, M. J. Longo, and R. A. Street, "Demonstration of megavoltage and diagnostic X-ray imaging with hydrogenated amorphous silicon arrays," Med. Phys. 19, 1455-1466 (1992).

${ }^{13}$ J. C. Dainty and R. Shaw, Image Science: Principles, Analysis and Evaluation of Photographic-Type Imaging Processes (Academic, London, 1974).

${ }^{14}$ M. L. Giger, K. Doi, and C. E. Metz, "Investigation of basic imaging properties in digital radiography. 2. Noise Wiener spectrum," Med. Phys. 11, 797-805 (1984).

${ }^{15}$ A. D. A. Maidment and M. J. Yaffe, "Analysis of the spatial-frequencydependent DQE of optically coupled digital mammography detectors," Med. Phys. 21, 721-729 (1994).

${ }^{16}$ J. T. Dobbins, D. L. Ergun, L. Rutz, D. A. Hinshaw, H. Blume, and D. C. Clark, " $\mathrm{DQE}(f)$ of four generations of computed radiography acquisition devices," Med. Phys. 22, 1581-1593 (1995).

${ }^{17}$ J. S. Bendat and A. G. Piersol, Random Data: Analysis and Measurement Procedures, 2nd ed. (J Wiley, New York, 1986).

${ }^{18}$ T. P. Krauss, L. Shure, and J. N. Little, Signal Processing Toolbox, For Use with Matlab (The Math Works, Inc., Natick, MA, 1994).

${ }^{19} \mathrm{R}$. VanMetter, "Linear systems techniques in imaging science," talk given at SPIE Physics of Medical Imaging workshop, February, 1996.

${ }^{20}$ I. A. Cunningham, "Analyzing system performance,"' in The Expanding Role of Medical Physics in Diagnostic Imaging, edited by G. D. Frey and P. S. Sprawls (Advanced Medical Publishing, Madison, WI, 1997).

${ }^{21}$ H. E. Johns and J. R. Cunningham, The Physics of Radiology (Thomas, Springfield, 1983).

${ }^{22}$ R. Birch, M. Marshall, and G. M. Ardran, "Catalogue of spectral data for diagnostic x-rays," Hospital Physicists' Association, Scientific Report Series 30, 1979, pp. 20-31

${ }^{23}$ M. Rabbani, R. Shaw, and R. Van Metter, "Detective quantum efficiency of imaging systems with amplifying and scattering mechanisms," J. Opt. Soc. Am. A 4, 895-901 (1987).

${ }^{24}$ J. H. Siewerdsen, L. E. Antonuk, and J. Yorkston, "Theoretical performance of amorphous silicon imagers in diagnostic radiology," Medical Imaging 1996: Physics of Medical Imaging, SPIE 2708, 1996, pp. 484493.

${ }^{25}$ I. A. Cunningham, M. S. Westmore, and A. Fenster, " A spatial-frequency dependent quantum accounting diagram and detective quantum efficiency model of signal and noise propagation in cascaded imaging systems," Med. Phys. 21, 417-427 (1994).

${ }^{26}$ I. A. Cunningham, M. S. Westmore, and A. Fenster, "Visual impact of the non-zero spatial frequency quantum sink," in Ref. 8, pp. 274-283.

${ }^{27}$ I. A. Cunningham, "Degradation of the detective quantum efficiency due to a non-unity detector fill factor," Medical Imaging 1997: Physics of Medical Imaging, SPIE 3032, 1997, pp. 22-31.

${ }^{28}$ R. L. Weisfield, R. A. Street, R. Apte, and A. Moore, "An improved page-sized $127 \mu \mathrm{m}$ pixel amorphous silicon image sensor for X-ray diagnostic medical imaging applications," in Ref. 27, pp. 14-21.

${ }^{29}$ F. G. Rueter, B. J. Conway, J. L. McCrohan, and O. H. Suleiman, "Average radiation exposure values for three diagnostic radiographic examinations," Radiology 177, 341-345 (1990).

${ }^{30}$ J. M. Boone, D. E. Pfeiffer, K. J. Strauss, R. P. Rossi, P. P. Lin, J. S. Shepard, and B. J. Conway, "A survey of fluoroscopic exposure rates: AAPM task Group No. 11 report,” Med. Phys. 20, 789-794 (1993).

${ }^{31}$ H. Wieczorek, G. Frings, P. Quadflieg, and U. Schiebel, "CsI:Tl for solid state x-ray detectors," Proceedings of the International Conference on Inorganic Scintillators and their Applications, Delft, Netherlands, 1995.

${ }^{32}$ R. M. Gagne, C. N. West, R. F. Wagner, and P. W. Quinn, "Laboratory 
measurements of sensitometry, MTF, veiling glare, Wiener spectrum and DQE for image intensifier tubes," Medical Imaging 1993: Physics of Medical Imaging, SPIE 1896, 1993, pp. 248-258.

${ }^{33}$ E. Storm and H. I. Israel, "Photon cross sections from $1 \mathrm{keV}$ to $100 \mathrm{MeV}$ for elements $Z=1$ to $Z=100$,' Nucl. Data Tables A 7, 565-681 (1970).

${ }^{34}$ J. A. Rowlands and K. W. Taylor, "Absorption and noise in cesium iodide x-ray image intensifiers,' Med. Phys. 10, 786-795 (1983)

${ }^{35}$ W. Hillen, W. Eckenbach, P. Quadflieg, and P. Zaengel, "Signal-to-noise performance in cesium iodide X-ray fluorescent screens," Medical Imaging V: Image Physics, SPIE 1443, 1991, pp. 120-131.

${ }^{36}$ R. K. Swank, "Absorption and noise in X-ray phosphors,' J. Appl. Phys. 44, 4199-4203 (1973).

${ }^{37}$ L. E. Antonuk, J. H. Siewerdsen, J. Yorkston, and W. Huang, ' Radiation response of amorphous silicon imaging arrays at diagnostic energies," IEEE Trans. Nucl. Sci. 14, 1500-1505 (1994).

${ }^{38}$ P. C. Bunch, K. E. Huff, and R. Van Metter, "Analysis of the detective quantum efficiency of a radiographic screen-film combination,' J. Opt. Soc. Am. A 4, 902-909 (1987).

${ }^{39}$ R. Shaw, "Quantifying the efficiency of imaging systems: A decade of progress in optimizing screen-films for x-ray detection,'” in Ref. 9, pp. $2-11$.

${ }^{40}$ J. M. Boudry and L. E. Antonuk, 'Radiation damage of amorphous silicon photodiode sensors,' IEEE Trans. Nucl. Sci. 41, 703-707 (1994).

${ }^{41}$ J. M. Boudry and L. E. Antonuk, "Radiation damage of amorphous silicon, thin-film, field-effect transistors,"' Med. Phys. 23, 743-754 (1996).

${ }^{42}$ W. Zhao and J. A. Rowlands, "X-ray imaging using amorphous selenium: Feasibility of a flat panel self-scanned detector digital radiology,', Med. Phys. 22, 1595-1604 (1995).

${ }^{43}$ D. L. Lee, L. K. Cheung, and L. S. Jeromin, "A new digital detector for projection radiography,' in Ref. 9, pp. 237-249.

${ }^{44}$ K. S. Shah, P. Bennett, M. Klugerman, L. P. Moy, G. Entine, D. Ouimette, and R. Aikens, "Lead iodide films for x-ray imaging," in Ref. 27, pp. 395-404.

${ }^{45}$ J. A. Rowlands, W. Zhao, I. Blevis, G. Pang, W. G. Ji, S. Germann, S. O. Kasap, D. Waechter, and Z. Huang, "Flat panel detector for digital radiology using active matrix readout of amorphous selenium,', in Ref. 27, pp. $97-108$. 